\title{
Mixed integer non-linear programming model for reliable and safer design at an early stage
}

\author{
Daniel Vázquez*, Rubén Ruiz-Femenia, José A. Caballero \\ Institute of Chemical Process Engineering, University of Alicante, PO 99, E-3080 Alicante, Spain
}

\section{A R T I C L E I N F O}

Article history:

Received 5 February 2020

Revised 13 January 2021

Accepted 5 February 2021

Available online 7 February 2021

\section{Keywords:}

Reliability

Process safety

Mixed integer non-linear programming

\begin{abstract}
A B S T R A C T
We present a Mixed Integer Non-Linear Programming (MINLP) model capable of choosing the best design considering economic profit, availability, and safety. The model takes into account the probability of suffering a failure in a year of operation, as well as the revenue generated and the probability of the process units of being in a non-functional state. The inclusion of programmed maintenances of a specified duration is considered in the model, assuming an equal distribution in the maintenances time. The performance of the model is illustrated by small examples to help the reader to better understand the model, before applying it to the methanol synthesis case study, where the economic and safety objectives are represented in a Pareto front. The results showcase the possibility of considering safety during the early design stage.
\end{abstract}

(c) 2021 Elsevier Ltd. All rights reserved.

\section{Introduction}

During the design of an industrial process, one of the more important considerations besides the obvious economic and environmental aspects, is the availability of the plant. This variable directly influences the two aforementioned aspects, in that if the plant is not available, there is no production, which has both an economic and an environmental impact. Usually, this is a negative impact on the profit, since less product is produced, and either a positive or a negative impact on the environmental objectives, since the unavailability can be produced by failures that result in dangerous spills. A possibility to evaluate the availability is the use of discrete event simulations that are able to evaluate design alternatives using historical maintenance data and statistical models (Sharda and Bury, 2008).

A certain field of engineering specializes in this concept: reliability engineering. It emphasizes the development of methods to evaluate and optimize reliability/availability in a quantitative manner. Zio (2009) states that reliability is a fundamental attribute for safety during operation in any modern technological system. Therefore, reliability analysis aims at obtaining the mathematical quantification of the probability of failure of both the studied system and its possible protective barriers.

An important difficulty of optimizing flowsheets considering the availability is the complexity that an industrial plant entails. There

\footnotetext{
* Corresponding author.

E-mail address: vazquez.vazquez@ua.es (D. Vázquez).
}

are multiple states possible for each unit. Some works address the effectivity of Markov chains on both statistical and analytical methods (Lin et al., 2012; Lisnianski et al., 2010). Other authors (H. Goel et al., 2003; Goel et al., 2003; H. D. Goel et al., 2003; Pistikopoulos et al., 2001) presented MILP models able of selecting different process units considering the reliability, production and maintenance planning for a fixed given configuration. However, up until recently, there was no general rigorous mixed-integer optimization model proposed that would systematically choose the correct design in order to obtain the optimal flowsheet both with the availability and the cost as main objectives. The work of Ye et al. (2018) succeeded in presenting a functional, elegant model, that allows the decision-maker to take into account availability when deciding the design of the plant.

Another important consideration for the design of an industrial plant is safety. In the same manner as the availability, it is not usually considered at the same level as the economic and environmental objectives during design. Historically, safety is a secondary consideration, calculated after the main design is already established. However, by doing this, some designs that may have been more balanced considering all the objectives are discarded (AIChE, 2010; Ordouei et al., 2014; D. Song et al., 2018). As a response, Inherent Safer Design (ISD), which is based on the work by Kletz and Amyotte (2010), aims to provide concepts that allow safety analysis to play a more important role during early design phases. The main philosophy behind these concepts is to consider safety as a design driving force instead of as an add-on. There are multiple works that take into account inherent safety, as well as many safety in- 
dices that start to incorporate these concepts (Jafari et al., 2018; Roy et al., 2016; Ruiz-Femenia et al., 2017). Some examples are the Hazard Identification and Ranking (HIRA) (Faisal I. Khan and Abbasi, 1998), I2SI (Faisal I. Khan and Abbasi, 1998), Quantitative Index of Inherently Safer Design (QI2SD) (Rusli et al., 2013), Optimizable Fuzzy Inherent Safety Index (OFISI) (Vázquez et al., 2019), etc. Even some indices not thought to perform as inherent safety indices, such as Dow's F\&EI (AIChE, 1994) and the Safety Weighted Hazard Index (SWeHI) (F. I. Khan et al., 2001) have been considered to work as such (Vázquez, et al., 2018b). These indices take into account properties of the process units, such as the amount of chemical inside, temperature and pressure of operation, and provide an estimation of the damage that unit could case if something malfunctioned. Another option is the use of risk analysis, which takes into account the probability of a failure occurring. This is done using the Chemical Process Quantitative Risk Analysis (CPQRA), which is a methodology designed to provide a tool that helps to evaluate overall process safety in the chemical process industry. However, these methodologies are based on given designs. This means, their utility when using them to choose an optimal option of design is limited by the size of branching possibilities that can be chosen. The number of combinations of possible designs makes studying each possible combination separately difficult.

Availability and safety are closely related (Houssin and Coulibaly, 2014; Yi et al., 2019). If a process unit is unavailable, it may be because it has suffered a failure, which in fact could cause damage. In the same manner, in order to increase the availability, a common solution is to have more than one copy of the same process unit, even if that spare unit will only be turned on when the main unit is not available. Therefore, it is expected that the safety level of the design will be reduced, since more availability means more time of operation for the units and therefore more probability of a failure happening.

In this paper, we extend the Mixed Integer Non-Linear model developed by Ye et al. (2018) to account for a safer design. We also introduce the possibility of adding maintenances to the operational year, which impact both the safety and the availability, and therefore, the expected profit. While this is technically not a scheduling model, such as the likes of Kondili et al. (1993) or AlMutairi et al. (2008) among others, since we do not consider either continues or discontinuous slots of time, nor if we have to start a machine at a set time in the process, the resultant model can provide the optimal time of work for each machine in the process during a year of operation, since it is clear that this operation time is closely related to both the availability and the safety objectives.

The paper is structured as follows: first, the base model from Ye et al. (2018) is presented, as well as the modifications performed, showing how some probabilities are calculated as well as a small example case to prove how it performs. The introduction of safety in this model is then presented, modifying the previous example case. Afterwards, the introduction of maintenance time and how to consider it is explained, as well as how to include it in the model. A case study is then showcased where the full model displays its capabilities. Finally, the conclusions of the work are presented.

\section{Problem statement}

At this point in time, a mathematical model able to choose the optimal design based on the availability and economic objectives already exists. The objective of this paper is to extend that said model to take into account not only the availability, but the reliability and safety as well. In order to do this, an MINLP model is formulated, where the following considerations are taken into account.
- The possibility of having multiple different machines able to perform the same work, where they present different availabilities, different capacities of work, different repairing times, etc..

- The highest the operation time of a single machine, the more probable it is that said machine suffers a failure. This goes against the safety objective. However, the highest the operation time, if there are not repeated machines, the highest is the resultant availability.

- The safety of the plant is not only compromised by the amount of machines operating, but their capacity. These two parameters result in a different safety objective for each configuration possible.

The model chooses which process units, from a pre-stated selection given, are installed, as well as the number of maintenances equally spaced in time should be performed in order to optimize one of three objectives, these being economic, availability and safety. The possibility of parallel units is contemplated, although limited in this case to full and half capacity machines. A full capacity machine is defined as a process unit such that its existence is enough to satisfy the constraints of production in the flowsheet, while a half capacity machine requires two units in parallel working at the same time to satisfy these constraints.

\section{Availability and probability of failure}

In order to make a system more reliable, increasing its availability is regarded as the most direct approach. A common method of increasing the total availability of a process is to use redundant units that only start working once the main unit has failed or is in maintenance. Another possibility is to use smaller redundant units, so the process may continue with half capacity while the main unit is unavailable. The base model takes into account the possibility of using both full and half capacity units. Different sizes for the units could also be considered, but the tree of possibilities would grow exponentially, resulting in the model being too complicated to be solved in a reasonable amount of time.

\subsection{Base model}

The model is based upon the model from Ye et al. (2018). We consider a set of stages, $k \in K$, and a set of parallel units, $j \in J$, as shown in (1).

$J:=\{j:$ Set of possible machines $\}$

$K:=\{k:$ Set of stages present in the superstructure $\}$

In each stage, there are a number of units that have full capacity and others that have half capacity. We define the sets $J F_{k, j}$ and $\mathrm{JH}_{k, j}$ as the set that correlates the machines at each stage that operate at full capacity and half capacity, as shown in (2).

$J F_{k, j}:=\{(k, j)$ : Machine $j$ in stage $k$ operates at full capacity $\}$

$J H_{k, j}:=\{(k, j)$ : Machine $j$ in stage $k$ operates at half capacity $\}$

We define $y_{k, j}$ as a binary variable that indicates if the machine $j$ is selected in stage $k$. The first constraint is the satisfaction constraint. There must be enough machines to at least fulfill the total capacity of the plant. This constraint is shown in Eq. (3).

$\sum_{j} C p_{k, j} y_{k, j} \geq 1 \quad \forall k \in K$

Where $C p_{k, j}$ is defined as:

$C p_{k, j}=\left\{\begin{array}{ll}1 & j \in J F_{j, k} \\ 0.5 & j \in J H_{j, k}\end{array} \quad \forall k \in K\right.$ 
Then, we must define the availability. The availability of a machine can be defined as its uptime. This means, the percentage of the time that the machine is working. We will refer to this concept using the parameter $p a_{k, j}$. It can be interpreted as the probability of the machine being in a working state at a determined period of time. An uptime of 0.99 means that it remains $99 \%$ of the time in a working state, which therefore has the probability of being in a not-working state of $1 \%$. This must not be mistaken by the probability of having a failure in a period of time.

We define a setting system for each stage. The settings are each of the possibilities of a stage, which correspond to each available combination of chosen machines. For example, if a stage $k$ has the following superstructure:

There are a total of $\left(\begin{array}{l}4 \\ 1\end{array}\right)+\left(\begin{array}{l}4 \\ 2\end{array}\right)+\left(\begin{array}{l}4 \\ 3\end{array}\right)+\left(\begin{array}{l}4 \\ 4\end{array}\right)=4+6+4+1=15$ different settings. We define a set of settings $s \in S$ that will be dynamically bounded by the set $K S_{k, s}$. This set shows how many settings are possible for a stage $k$. Both these sets are defined as shown in (5)

$S:=\{s:$ Set of possible settings $\}$

$K S_{k, s}:=\{(k, s)$ : Setting $s$ is in stage $k\}$

Another set, $J S K_{k, s, j}$, specifies which machines are present in each setting for each stage (6).

$J S K_{k, s, j}:=\{(k, s, j):$ Machine $j$ is present in setting $s$ in stage $k\}$

This set is dynamically generated with the help of a parameter $\alpha_{k, s, j}$ which is calculated from Eq. (7)

$\alpha_{k, s, j}=\left\lfloor\frac{\bmod \left(s, 2^{j}\right)}{2^{j-1}}\right\rfloor \forall k, s, j \in J S K_{k, s, j}$

If $\alpha_{k, s, j}=1$, that means that in that $k$ stage, in that $s$ setting, machine $j$ exists. We define a binary variable $z_{k, s}$ which takes the value of 1 if we are at a certain setting in a certain stage, and 0 otherwise. This binary is related to the binary variables $y_{k, j}$ by the following logical propositions in terms of their corresponding Boolean variables. Even though the meaning of Boolean and binary variables is not the same, for the sake of clarity, we use the same symbol for both. In any case, there is a one-to-one relationship between the Boolean and binary variable that avoid any misunderstanding that this abuse in notation could produce. These relationships are shown in Eq. (8).

$z_{k, s} \Rightarrow y_{k, j} \quad \forall k, s, j \in J S K_{k, s, j}$

$z_{k, s} \Rightarrow \neg y_{k, j} \quad \forall k, s, j \notin J S K_{k, s, j}$

$\underline{\vee}_{s \in K S_{k, s}}\left(z_{k, s}\right) \quad \forall k \in K$

Which can be transformed into algebraic expressions (Grossmann and Trespalacios, 2013), turning into Eq. (9).

$\begin{array}{ll}z_{k, s} \leq y_{k, j} & \forall k, s, j \in J S K_{k, s, j} \\ y_{k, j}+z_{k, s,} \leq 1 & \forall k, s, j \in K S_{k, s} \notin J S K_{k, s, j} \\ \sum_{s \in K S_{k, j}} z_{k, s}=1 & \forall k \in K\end{array}$

Ye et al. (2018) divided the availability between the availability at half capacity and the availability at full capacity. These are defined by the probability of a stage to be at full capacity or half capacity. For a stage to be at half capacity, the main condition is that all its full capacity units have failed. Then, we have to take into account the possibilities of each half capacity unit working alone, this means, the other half capacity units have failed. This can be extended to each possible setting for each stage. We define the probability of working at half capacity in a stage $k$ at a setting

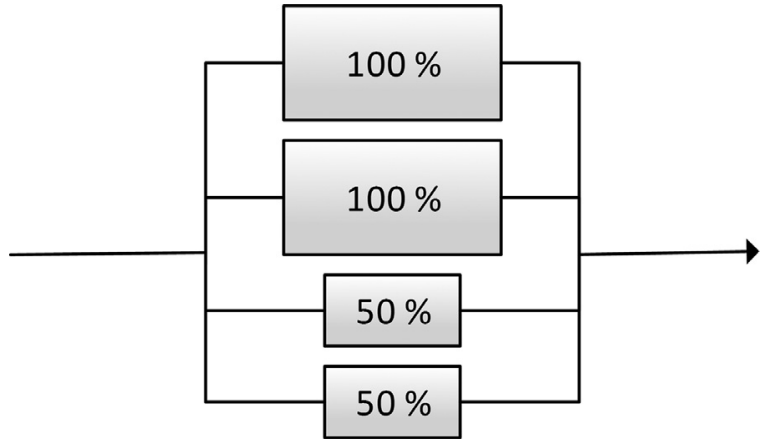

Fig. 1. Example of a superstructure of settings in a stage $k$ with two full capacity machines and two half capacity machines.

$s$ as $P s_{k, s}^{H}$. It is obtained from Eq. (10).

$$
\begin{aligned}
P S_{k, s}^{H}= & \prod_{j \in J F_{j, k}, J S K_{k, s, j}}\left(1-p a_{k, j}\right) \times\left[\sum_{j \in J H_{j, k}, J S K_{k, s, j}} p a_{k, j} \cdot \prod_{\substack{j^{\prime} \in J H, J S K \\
j^{\prime} \neq j}}\left(1-p a_{k, j^{\prime}}\right)\right] \\
& \times z_{k, s} \quad \forall k, s \in K S_{k, s}
\end{aligned}
$$

For example, for a stage equal to the one depicted in Fig. 1, for a setting that considers that these four units are present, the probability of working at half capacity would be obtained as shown in Eq. (11).

$P s^{H}=\left(1-p a_{1}\right)\left(1-p a_{2}\right)\left[p a_{3}\left(1-p a_{4}\right)+p a_{4}\left(1-p a_{3}\right)\right]$

Of course, only one setting can be active at each stage, which is why the binary $z_{k, s}$ appears multiplying, so in order to obtain the probability of working at half capacity, we use Eq. (12).

$P_{k}^{H}=\sum_{s \in K S_{k, s}} P s_{k, s}^{H} \quad \forall k \in K$

The probability of working at full capacity in each setting, $P s_{k, s}^{F}$, can be obtained from the probability of working at half capacity. There are only three possibilities for each setting. Either it is working at full capacity, half capacity or not working at all. Therefore, we can define the probability of working at full capacity from the probability of total failure and the probability of working at half capacity. The equation is shown in Eq. (13).

$P s_{k, s}^{F}=\left[1-\prod_{j \in J F_{j, k}, J H_{j, k}}\left(1-p a_{j, k}\right)-P s_{k, s}^{H}\right] z_{k, s} \quad \forall k, s \in K S_{k, s}$

As in the case of the probability of half capacity, only one setting for stage will be selected. So, the probability of working at full capacity in a stage $k$ is as shown in Eq. (14).

$P_{k}^{F}=\sum_{s \in K S_{k, s}} P s_{k, s}^{F} \quad \forall k \in K$

The availability at half capacity, full capacity, and the general availability are defined as shown in Eq. (15)

$A^{F}=\prod_{k \in K} P_{k}^{F}$

$A^{H}=\prod_{k \in K}\left(P_{k}^{F}+P_{k}^{H}\right)-A^{F}$

$A=A^{F}+\frac{A^{H}}{2}$

Regarding the availability, a penalty and a benefit are considered, since normally the plant and the customer establish a contract, with two reference limits set for the availability. If the availability is between the bounds, there is no penalization nor benefit 


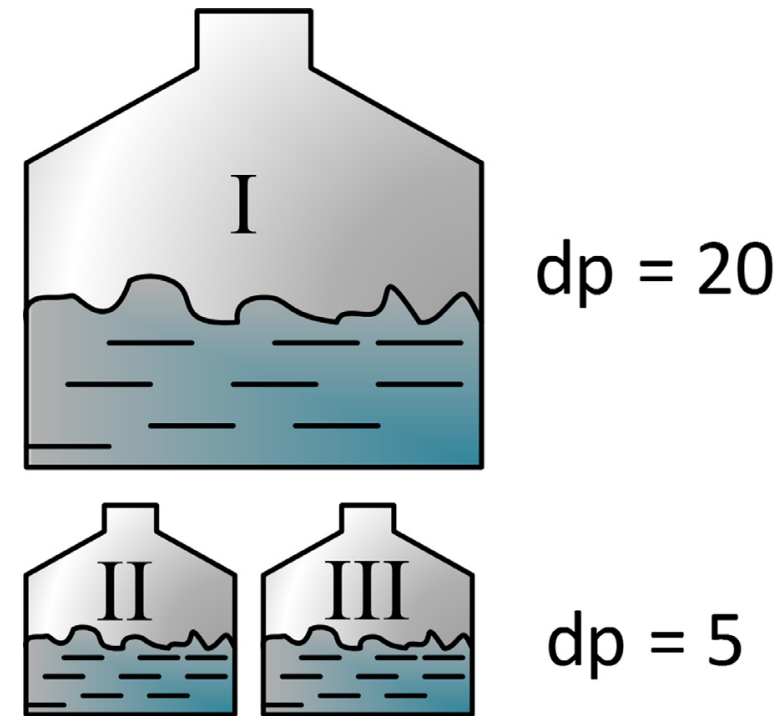

Fig. 2. Example for the addition of safety measurements.

associated. If it is below the lower bound, a linear penalization is considered, and if it is over the upper bound, a linear benefit is granted. This can be modeled by the disjunction shown in Eq. (16).

$\left[\begin{array}{c}v_{1} \\ A \leq A^{\text {low }} \\ B n^{A}=0 \\ \operatorname{Pen}^{A}=f \operatorname{pen}^{A}\left(A^{\text {low }}-A\right)\end{array}\right] \unrhd\left[\begin{array}{c}v_{2} \\ A^{\text {low }} \leq A \leq A^{\text {high }} \\ B n^{A}=0 \\ \operatorname{Pen}^{A}=0\end{array}\right] \vee\left[\begin{array}{c}v_{3} \\ A \geq A^{\text {high }} \\ B n^{A}=f \operatorname{ben}^{A}\left(A-A^{\text {high }}\right) \\ \operatorname{Pen}^{A}=0\end{array}\right]$

After applying the convex hull reformulation (Lee and Grossmann, 2000), this disjunction results in the algebraic equations shown in Eq. (17).

$$
\begin{aligned}
v_{1}+v_{2}+v_{3} & =1 \\
A & =A_{1}+A_{2}+A_{3} \\
B n^{A} & =\left(A_{3}-A^{\text {high }} v_{3}\right) \text { fben }^{A} \\
\operatorname{Pen}^{A} & =\left(A^{l o w} v_{1}-A_{1}\right) \text { fpen }^{A} \\
A_{1} & \leq A^{\text {low }} v_{1} \\
A^{\text {low }} v_{2} & \leq A_{2} \leq A^{\text {high }} v_{2} \\
A^{\text {high }} v_{3} & \leq A_{3} \leq v_{3}
\end{aligned}
$$

The objective function accounts for these penalizations and benefits, and the cost of the equipment. Ye et al. (2018) divided the cost between installation and reparation cost, such that the cost of a stage $k$ is defined by Eq. (18).

$C_{k}=\sum_{j} y_{k, j}\left(c_{k, j}^{i n s t}+c_{k, j}^{r e p}\right) \quad \forall k \in K$

The positive contribution to the objective function arises from the expected revenue. For this, a parameter $r v$ that states the revenue of the plant is used, as shown in Eq. (19).

$E R V=r v \times A$

An initial objective function is then shown in Eq. (20).

$\max z=E R V-P e n^{A}+B n^{A}-\sum_{k \in K} C_{k}$

\subsection{Addition of safety measurements}

Regarding the measure of safety, we consider a probabilistic approach. Let us consider a simple example case, shown in Fig. 2.

Where $d p$ stands for damage potential. Unit I has a higher damage potential than units II and III, since its size and amount of chemical inside is bigger. The damage potential can be measured using different indices, such as Dow's F\&EI. These indices are normally not linear with the size of the process unit. Let us assume that the probability of one of these units failing in a year of operation is $20 \%$. We define the damage as a risk tree, taking into account the damage of each possible output. For example, here, we have the following possibilities:

- Only one fails. The damage would be then be calculated as the damage of each failure, multiplied by the probability of that failure happening while the other units do not fail. In this case the result would be 3.84 .

- Two fail. The damage would be then calculated as the damage of each subset of size two possible, which for this case are $\{I, I I\},\{I, I I I\},\{I I, I I I\}$, multiplied for the possibility of each of these subsets happening. In this case, the result is 1.92 .

- All three fail. The damage is the damage of the only subset of size three, $\{I, I I, I I I\}$, multiplied by the possibility of this subset happening. The result is 0.24 .

Therefore, the expected damage of this setting would be $3.84+1.92+0.24=6.0$. The equation that strictly defines this is shown in Eq. (21).

Damage $_{k, s}=\left[\sum_{i} \sum_{j \in K S I J_{k, s, i, j}} d p_{k, j} \prod_{j \in K S I J_{k, s, i, j}} p f_{k, s, j} \prod_{\substack{j \in J S K_{k, s, j} \\ j \notin K S I J_{k, s, i, j}}}\left(1-p f_{k, s, j}\right)\right] z_{k, s}$

Where $i$ is a set that enumerates the subsets in each setting $s$ in each stage $k$, therefore, is another nested calculation of subsets, similar to the ones calculated with the parameter $\alpha_{k, s, j}$. Luckily for us, there is another way to obtain this damage using the concept of expected value. The expected value, or the mean value, of a random variable with finite realizations is defined as shown in Eq. (22).

$E[X]=\sum_{i} x_{i} p_{i}$

For example, the expected value of the damage for the first unit process I, supposing a probability of failure of $20 \%$, would be $E\left[X_{I}\right]=20 \times 0.2+0 \times(1-0.2)=4$. An important property of the expected value is that it is a linear operator, and hence, it follows the formula shown in Eq. (23).

$E\left[X_{1}+X_{2}\right]=E\left[X_{1}\right]+E\left[X_{2}\right]$

Therefore, for our example, the result would be as shown in Eq. (24).

$$
\begin{aligned}
& E\left[X_{I}+X_{I I}+X_{I I I}\right] \\
& \quad=(20 \times 0.2+0 \times 0.8)+(5 \times 0.2+0 \times 0.8)+(5 \times 0.2+0 \times 0.8)=6
\end{aligned}
$$

Therefore, in the model, instead of Eq. (21) we use Eq. (25).

Damage $_{k, s}=\left[\sum_{j \in J K_{k, s, j}} d p_{k, j} p f_{k, s, j}\right] z_{k, s} \quad \forall k, s \in K S_{k, s}$

The remaining parameter needed is the probability of failure. For this, we assume that the probability of failure follows an exponential distribution with rate parameter $\lambda$. The probability of having a failure in an interval of time $\left[t_{0}, t\right]$ where $t_{0}=0$ is given by Eq. (26).

$p f=1-e^{-\lambda t}$

However, the operation time for each machine, $t$, depends on which stage, setting and machine we are looking into. It also depends on the order policy. We state that first, the full capacity machines are working, and once they fail, the half capacity machines 
operate the remaining time at an equal rate among them. This simplifies the model, which would have to consider a whole hierarchy of possible half capacity machines otherwise. Therefore, for the full capacity machines, considering a total operation time of $t^{\text {total }}$, the operation time $t^{o p}$ is obtained as shown in Eq. (27).

$$
t_{k, s, j}^{o p}=t^{\text {total }} p a_{k, j} \prod_{\substack{j^{\prime}<j \\ j^{\prime} \in J S K_{k, s, j} \\ j^{\prime} \in J F_{j, k}}}\left(1-p a_{k, j^{\prime}}\right) \quad \forall k, s, j \in J F_{j, k} \in J S K_{k, s, j}
$$

For half capacity machines, we have to take into account the number of subsets of $j$ half capacity machines being paired in each setting and stage. We also have to take into account if in the setting there is only one or more machines at half capacity. If there is only one, it will work all the operation time left by the full capacity machines. If there are two or more, all the subsets of size two will work the same amount of time. This means that if we have three half capacity machines, letś say $\mathrm{m} 1, \mathrm{~m} 2$, and $\mathrm{m} 3$, all the subset of size 2 (m1, m2), (m1, m3), (m2, m3) will work an equal amount of time. We define two sets, J1 and J2 which are defined in Eq. (28).

$J 1_{k, s}$ :

$=\{(k, s)$ : There is only one half capacity machine in the setting $s$ stage $k\}$ $J 2_{k, s}:$

$=\{(k, s):$ There are more than one half capacity machine in the setting $s$ stage $k\}$

We need to define two parameters to simplify the equations. We define $N S_{k, s}$ as the number of sets of size 2 of half machines present in a setting at a stage, and $\mathrm{NH}_{k, s}$ as the number of machines of half capacity present at a stage. They are determined by Eq. (29).

$\begin{aligned} N H_{k, s} & =\sum_{j \in J H_{j, k}, J S K_{k, s, j}} 1 \quad \forall k, s \in K S_{k, s} \\ N S_{k, s} & =\frac{N H_{k, s} !}{2 !\left(N H_{k, s}-2\right) !} \quad \forall k, s \in K S_{k, s}, J 2_{k, s}\end{aligned}$

Therefore, we get the following operation time both for the full machines and half machines at each stage and setting (Eq. (30)).

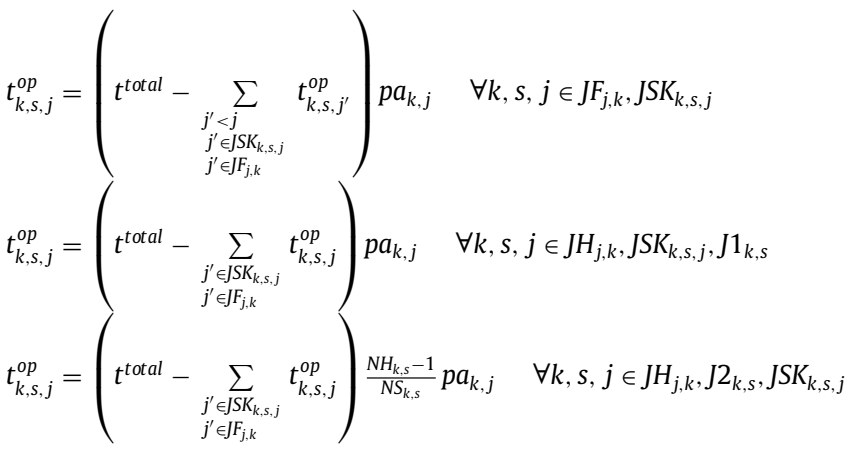

The damage of each stage is then calculated by Eq. (31).

$D_{k}=\sum_{s \in K S_{k, s}}$ Damage $_{k, s} \quad \forall k \in K$

And the expected damage of the design is obtained by Eq. (32).

$E D=\sum_{k \in K} D_{k}$

If we want to penalize the safety in the objective function, we can do it linearly with a parameter $P e n^{S}$. The objective function would then be to maximize the expected net profit (ENP) as shown in Eq. (33).

$E N P=E R V-P e n^{A}+B n^{A}-\sum_{k \in K} C_{k}-P e n^{S} E D$
Table 1

Installation costs for the example problem.

\begin{tabular}{llll}
\hline Stage/Machine & Machine 1 & Machine 2 & Machine 3 \\
\hline Stage 1 & 15 & 9 & 8 \\
Stage 2 & 5 & 3 & 2 \\
\hline
\end{tabular}

Table 2

Reparation costs for the example problem.

\begin{tabular}{llll}
\hline Stage/Machine & Machine 1 & Machine 2 & Machine 3 \\
\hline Stage 1 & 0.040 & 0.024 & 0.022 \\
Stage 2 & 0.032 & 0.030 & 0.028 \\
\hline
\end{tabular}

The full model consists of Eqs. (3), (9), (10), (12), (13), (14), (15), (17), (18), (19), (25), (31), (32) maximizing (33). We solve an example problem in order to show the versatility and possibility of the model. A depiction of the example is shown in Fig. 3.

The order of operation, as stated previously, is first $100 \%$ capacity units, and then $50 \%$ capacity units, where the $50 \%$ pairs of units are used an equal amount of time. The penalization parameter for the safety is $\mathrm{Pen}^{S}=0$. The limits for the availability are $A^{\text {low }}=0.988, A^{\text {high }}=0.996$. The costs are shown in Tables 1 and 2 . The rest of the parameters considered for this case example are: $r v=120, \mathrm{Pen}^{A}=130, B n^{A}=130$.

The results are shown in Figs. 4, 5 and 6.

As it is expected, the result of maximizing the availability will always be the alternative where every stage works with the setting containing the highest possible number of units. However, both the economic and safest results can vary depending on the price of the units, the availability of these units, and the damage potential of each unit.

\section{Introducing maintenance time}

\subsection{Probability distributions}

Up until now, we have considered a constant failure rate. This is true for the exponential distribution, which has the following probability density function (Eq. (34)).

$\operatorname{PDF}^{\exp }(x, \lambda)= \begin{cases}\lambda e^{-\lambda x} & x \geq 0 \\ 0 & x<0\end{cases}$

Therefore, the cumulative distribution function is as shown in Eq. (35).

$\operatorname{CDF}^{\exp }(x, \lambda)=\int \operatorname{PDF}^{\text {exp }}(x, \lambda) d x=e^{-\lambda x_{0}}-e^{-\lambda x}$

A characteristic of the exponential distribution is that it is memoryless. This means that any intention of performing maintenance between intervals of work will not result in an advantage. For example, consider a case with a constant rate of failure of $\lambda=1$. We consider an instant maintenance, which is a very ideal case, which would reset the time of error to 0 . This case is shown in Fig. 7. If we now calculate the possibility of at least one error happening for both zero and one maintenances, we get to the results in Eq. (36).

$P(\text { fail })^{\text {Zero }}=1-e^{-1 \times 5}=0.99326$

$P(\text { fail })^{\text {One }}=1-P(\neg$ fail $)=1-\left(e^{-1 \times 2.5}\right)\left(e^{-1 \times 2.5}\right)=0.99326$

The probability of not failing in an interval $\{0, t\}$ is $P\left(\right.$ fail $\left.^{\prime}\right)=$ $1-\left(1-e^{-\lambda t}\right)=e^{-\lambda t}$. Therefore, if we consider intervals $t_{0}, t_{1}$ such that they sum the initial interval $t$, the probability of the machine not failing would be the same as the initial interval, $P\left(\right.$ fail $\left.^{\prime}\right)=$ $e^{-\lambda t_{0}} e^{-\lambda t_{1}} \ldots e^{-\lambda t_{n}}=e^{-\lambda \sum t_{i}}=e^{-\lambda t}$. Having a number of real noninstantaneous maintenances would reduce the probability of failure, but only due to the fact that it would reduce the operation 


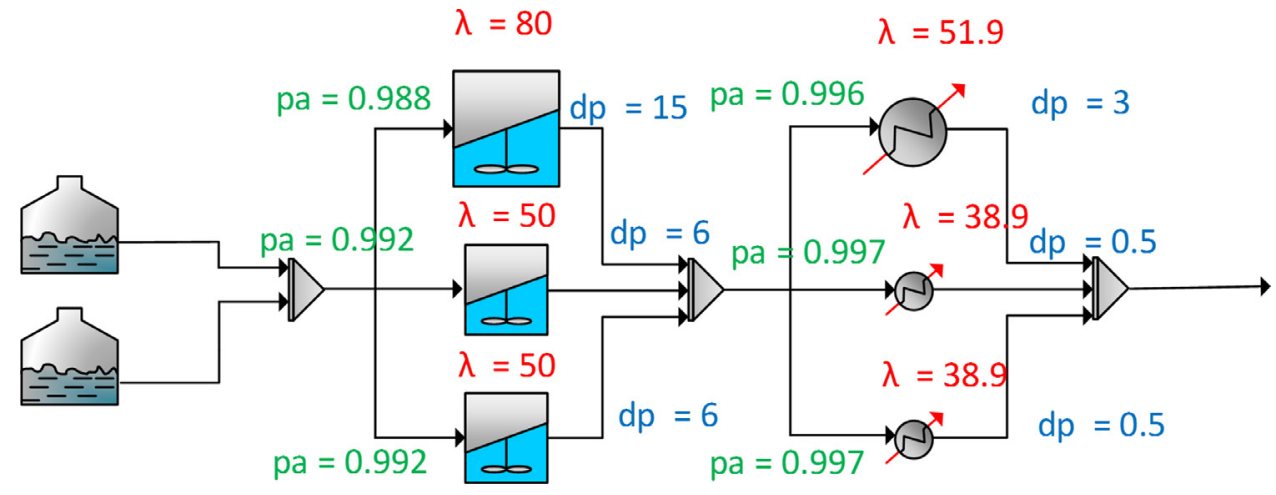

Fig. 3. Example problem flowsheet.

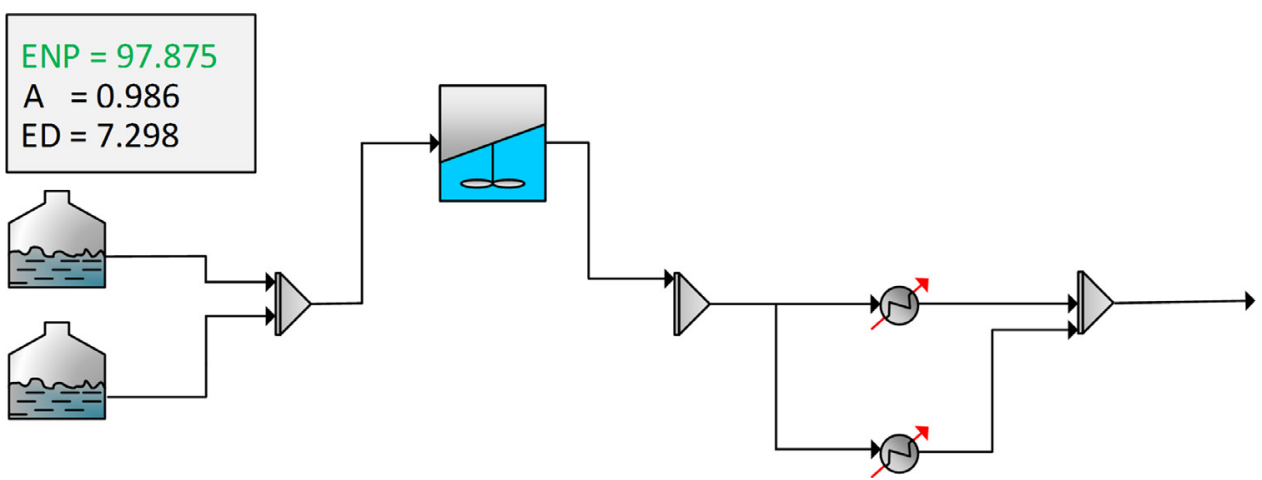

Fig. 4. Results of optimizing profit in the example problem.

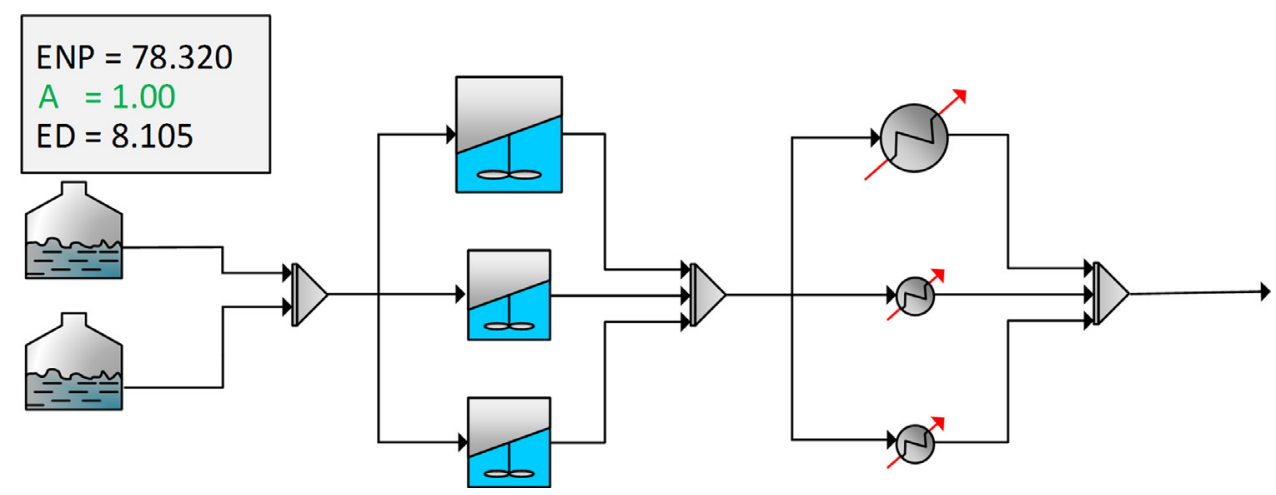

Fig. 5. Results of optimizing availability in the example problem.

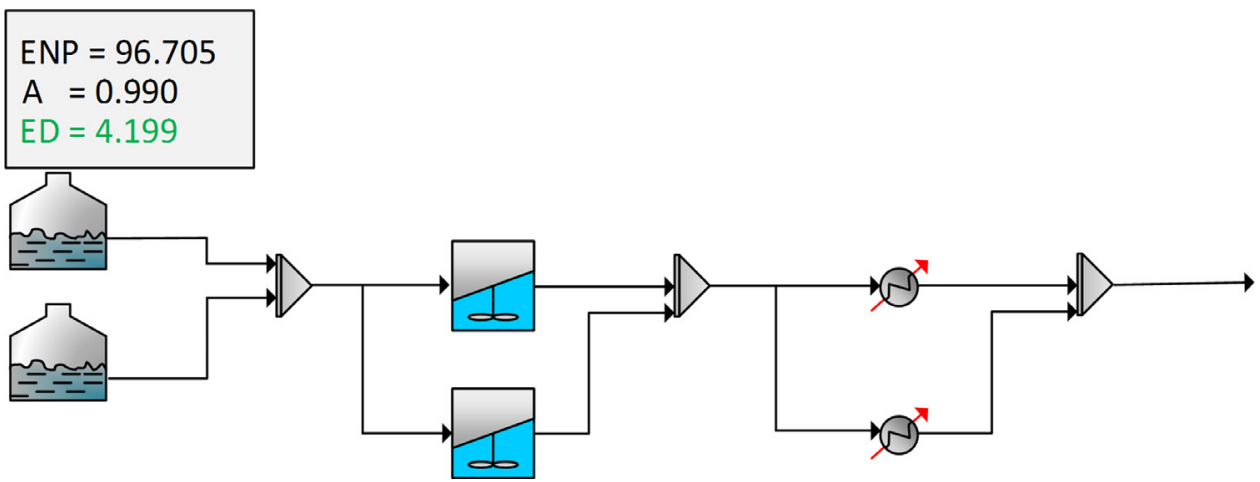

Fig. 6. Results of optimizing safety in the example problem. 


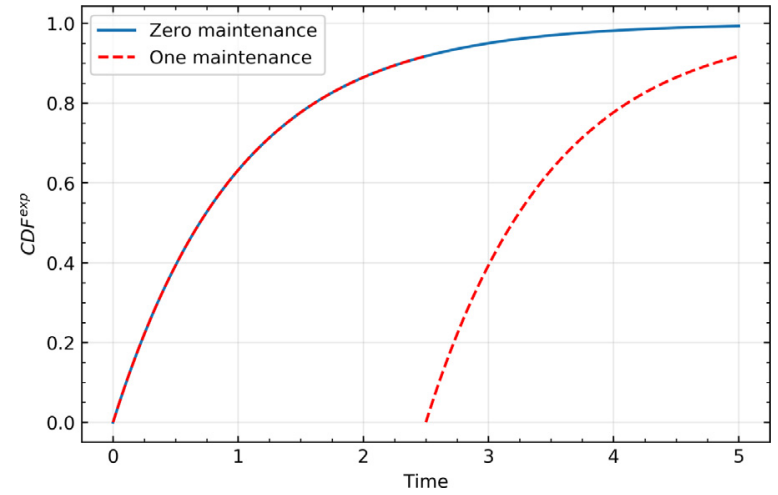

Fig. 7. Example case for maintenance in exponential distribution.

time. This is an intuitive idea. If the failure rate is supposed to be constant, it does not matter that maintenances are performed, since it will start from a point with the same failure rate. This is not a realistic concept, since it is expected that the functioning time of a piece of equipment will affect the probability of that unit failing.

Another common probability distribution used in reliability engineering is the Weibull distribution. Its probability density function is as shown in Eq. (37).

$\operatorname{PDF}^{\text {Weib }}(x, k, \theta)= \begin{cases}\frac{k}{\theta}\left(\frac{x}{\theta}\right)^{k-1} e^{-x / \theta^{k}} & x \geq 0 \\ 0 & x<0\end{cases}$

Its cumulative density function is as shown in Eq. (38).

$C D F^{W e i b}(x, k, \theta)=1-e^{-(x / \theta)^{k}}$

When $k=1$, if $\theta=1 / \lambda$, the Weibull distribution acts like an exponential distribution with parameter $\lambda$. However, at different values, its behavior is strongly dependent on the value of $k$. There are three possibilities:

$-k<1$ : This means that the failure rate decreases with $x$.

- $k=1$ : Exponential distribution, which means constant failure rate.

$-k>1$ : The failure rate increases with $x$. This is the expected case for process units.

Up until now, we have considered the failure rate to be constant and equal to $\lambda$. This is true for the exponential distribution. However, this is not true for the case of the Weibull distribution. The failure rate is linked to the hazard rate. The definition of hazard rate is shown in Eq. (39)

$h(t)=\frac{P D F(t)}{R(t)}$

Where $R(t)$ refers to the survival function, also called the reliability function. The survival function gives the probability of a unit surviving a given period of time. It is the complementary of the cumulative distribution function (Eq. (40))

$R(t)=1-F(t)$

Therefore, for the exponential distribution, the hazard rate is defined by Eq. (41).

$h(t)=\frac{\lambda e^{-\lambda x}}{1-\left(1-e^{-\lambda x}\right)}=\lambda$

For the Weibull distribution, the hazard rate is as shown in Eq. (42)

$h(t)=\frac{k}{\theta}\left(\frac{t}{\theta}\right)^{k-1}$

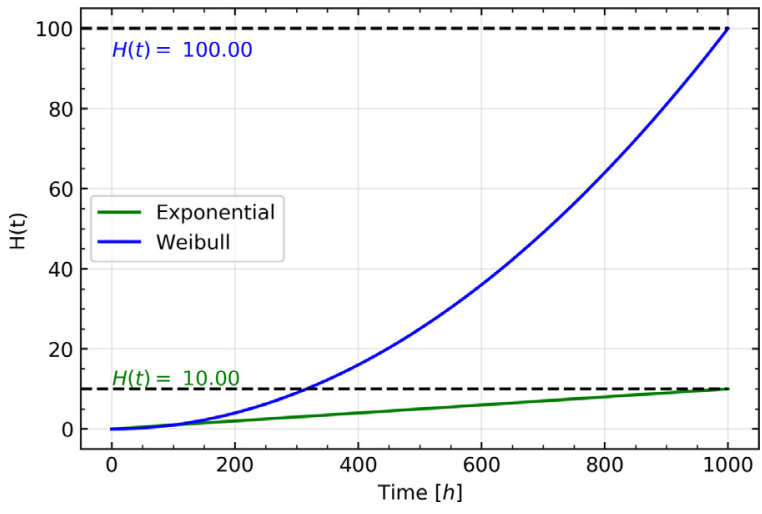

Fig. 8. Weibull and exponential cumulative hazard rate comparison.

The failure rate is defined from the hazard rate. Let us imagine that it is recorded that certain unit fails 10 times in 1000 hours. This failure rate, which is usually defined by $\lambda$ but since in this paper that is already the parameter of the exponential distribution we will use the letter $\Phi$, would be stated as:

$\Phi=\frac{10}{1000} \frac{\text { failures }}{\text { hour }}$

This is also defined by the hazard rate as shown in Eq. (43).

$\Phi \times t=H(t)=\int_{0}^{t} h(t) d t$

Where $H(t)$ is the cumulative hazard function, or the number of failures expected for a given interval of time. Of course, for the exponential distribution, $\lambda=\Phi$, however, that is not the case for other distributions, such as Weibull's. Let's suppose that we have a Weibull distribution with a value of $k=2$. First idea could be, similarly to the case for $k=1$, to consider $\theta=1 / \lambda$. However, if we look at the cumulative failure rate, we see that this is not true. Using the values given before, considering $\lambda=10 / 1000$ we obtain the figure shown in Fig. 8.

From the figure, it can be clearly seen that both distributions result in a different number of failures in the given time interval. If we want to ensure that the cumulative hazard rate is equal, once we set $k$ we can obtain $\theta$ by simply recalculating as shown in Eq. (44).

$$
\begin{aligned}
H(t)^{\text {Weib }} & =\int h(t) d t=\int \frac{k}{\theta}\left(\frac{t}{\theta}\right)^{k-1} d t=\frac{1}{\theta^{k}} t^{k} \\
\theta^{k} & =1 / H(t)^{\text {Weib }} t^{k}=\left(1 / 10 \times 1000^{2}\right)^{1 / 2} \approx 316
\end{aligned}
$$

If we use this parameter (Fig. 9), we see that the number of failures at the end of the interval remain the same.

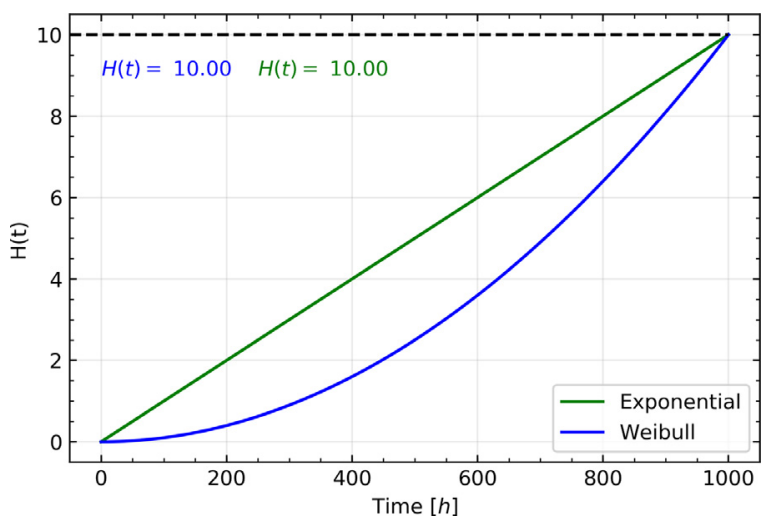

Fig. 9. Adjusted Weibull and exponential cumulative hazard rate. 

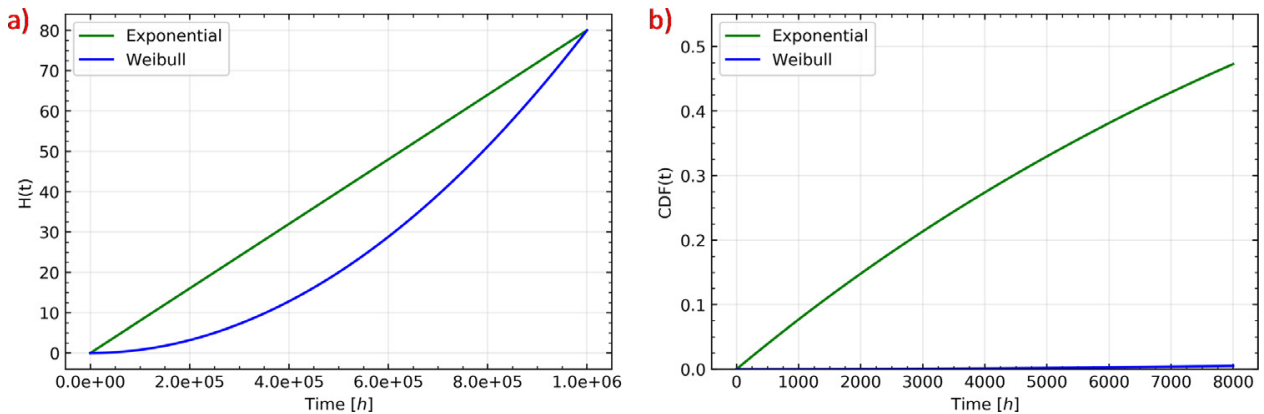

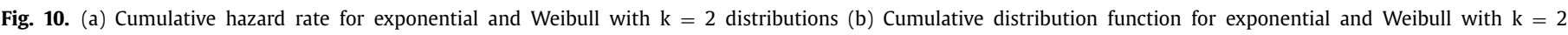
distributions.
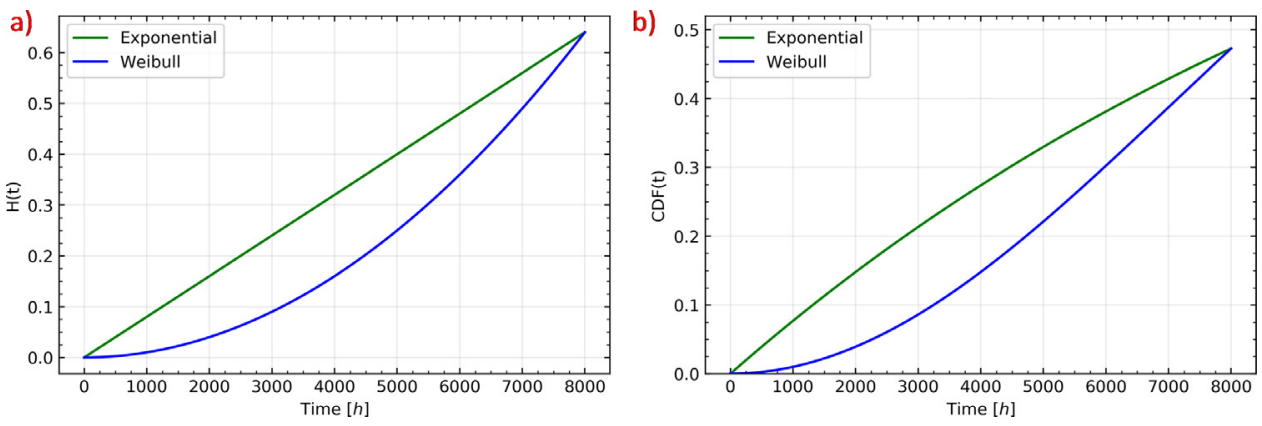

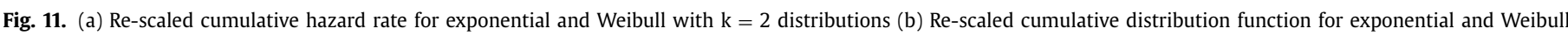
with $\mathrm{k}=2$ distributions.

This is not usually a problem when we know the failure distribution. This means that we can fit the failures and at which time they were produced to a function of time. However, in this paper, the only information provided is the raw number of failures in an interval of a million hours (AIChE, 1989). We are reducing the operation time to a yearly interval, so we have to be especially careful when supposing distributions other than the constant failure rate exponential distribution. As an example, suppose that we consider a unit that fails 80 times in a million hours. The equivalent Weibull distribution for $k=2$ is shown in Fig. 10.

If we take into consideration this distribution, the results when downgrading to a year will be immensely different. Therefore, even if it is not strictly true, considering that this is the only data we have, we scale everything with constant rate to a year and then calculate the appropriate distribution. Again, this is due to the fact that we lack the reliability data needed to fit a correct distribution, but we want to display the ability of the model to take into account different kinds of distributions. The re-scaled functions are shown in Fig. 11.

Using this scaled distribution, it is clear that with more maintenances, the probability of failure is reduced.

\subsection{Model}

Above, we defined the sets $k \in K, j \in J, s \in S$ as well as the relations $K S_{k, s}, J F_{j, k}, J H_{j, k}, J S K_{k, s, j}$. We now define a new set, $M M_{k, s, j}$, that will indicate at each stage $k$, setting $s$, which machines $j$ act as "main machines". Therefore we define the set as in (45).

$M M_{k, s, j}$ :

$=\{(k, s, j): j$ is a main machine in the setting $s$ at the stage $k\}$

The concept of main machines was explored above as in the order of operation. We consider that, for the stages where one or more full capacity machines are present, the main machine is the first of the full capacity machines. For stages where there are no full capacity machines, all the half capacity machines are considered as main machines, since they all will work the same amount of time. This means that we work following an strict order of operation where, in order for a unit to be active, all the units with higher priority have already failed, and all the half capacity machines share the same priority, while the priority of the full capacity machines is higher than that of the half capacity machines, and given by $j$.

The main machines are the only ones where we consider including maintenance. This is because the non-main machines do not work a significant amount of time yearly. Even for longer periods, the maintenance of these non-main machines could be performed during their off-time, when the main machines are working.

We define this dynamic set by the set of equations in Eq. (46)

$\begin{aligned} M M_{k, s, j} & =\left\{\begin{array}{ll}(k, s, j) \mid & \sum_{\substack{j^{\prime}<j \\ j^{\prime} \in J S K_{k, s, j^{\prime}} \\ j^{\prime} \in F_{j^{\prime}, k}}} 1=0 \quad \forall k, s, j \in K S_{k, s}, J F_{j, k}, J S K_{k, s, j}\end{array}\right\} \\ M M_{k, s, j} & =\left\{\begin{array}{l}(k, s, j) \mid \sum_{\substack{j^{\prime} \in J S K_{k, s, j^{\prime}} \\ j^{\prime} \in J F_{j^{\prime}, k}}} 1=0 \quad \forall k, s, j \in K S_{k, s}, J H_{j, k}, J S K_{k, s, j}\end{array}\right\}\end{aligned}$

We have to define the maintenance time. In Eq. (30), we computed the operation time from the availability of each machine. Now, in order to obtain the availability, we require some probabilistic states.

There are multiple ways of defining this. We choose the following. For a single machine, there are two main exclusive states. Either it is in maintenance or it is not. If it is not in maintenance, it has other two possible exclusive states. Either it is working, or it is in repair time. This can be represented using an Euler diagram, shown in Fig. 12.

Let us show a small example for the sake of clarity. Suppose that we have 10 hours of work. The maintenance time is 2 hours, therefore, the probability of maintenance is $P(M)=2 / 10$. Maintenance is an exclusive state with repairing (i.e., it cannot be repairing and in maintenance at the same time). Therefore, let's consider 


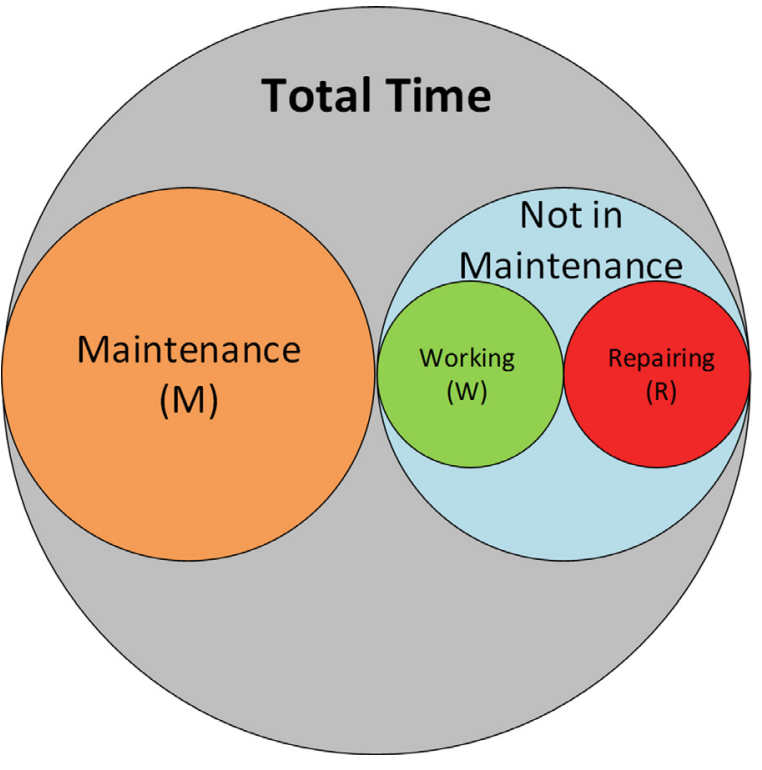

Fig. 12. Euler diagram for a single machine.

that the failing time, this being the amount of time that it remains in a failure state, is for example a tenth of the time that it is operating. This means that the probability of being in a failure / repairing state $(P(R))$, given that we are not in a maintenance state, is a tenth. This is written as $P\left(R \mid M^{\prime}\right)=1 / 10$. From this, using Kolmogorov definition to compute the conditional probability, we can obtain the probability of being in a state of repairing and not in maintenance, as shown in Eq. (47).

$P\left(R \cap M^{\prime}\right)=P\left(M^{\prime}\right) P\left(R \mid M^{\prime}\right)=(1-2 / 10)(1 / 10)=8 / 100$

As extracted from the Euler diagram, the probability of it being in repairing and not in maintenance is equal to the probability of it being in repairing. This is due to the fact that the state "Being in repairing" is completely contained within "Not in maintenance". From this diagram, it is clear as well that the probability of not working is equal to the probability of being in maintenance plus the probability of being in repairing. Therefore, the probability of being in a working state $(P(W))$ is calculated as in Eq. (48).

$P(W)=1-[P(M)+P(R)]=1-(2 / 10+8 / 100)=0.72$

Hence, availability is equal to 0.72 . This means that we can expect that this machine works $7.2 \mathrm{~h}$. In order to check the validity of this result, we now do the calculations considering time periods. If it works $10 \mathrm{~h}$, and the maintenance takes out $2 \mathrm{~h}$, there are $8 \mathrm{~h}$ remaining of work that are susceptible of having a failure. We considered that the probability of being in repairing state is $10 \%$ of the working time, which is $8 \mathrm{~h}$. Therefore, it is $0.8 \mathrm{~h}$ in a repairing state. The amount of time working is then $10-2-0.8=7.2 \mathrm{~h}$, resulting in an availability of 0.72 . It agrees with what we expect.

In summary, we consider the following assumptions:

- The states of working, repairing and maintenance are mutually exclusive and exhaustive.

- The probability of failing only applies to the time expected to work, this being, the time of operation minus the time of maintenance.

If there is no maintenance, there are only two possibilities. Either it is working or it is in a repair state. In order to calculate the probability of being in a repairing state, we use the concept of Mean Time Between Failures (MTBF). This is defined by Eq. (49).

$M T B F=\int_{0}^{\infty} R(t) d t$
If we assume a mean time between failures for the exponential distribution, the result is a constant, as shown in (50).

$\operatorname{MTBF}^{\text {exp }}=\int_{0}^{\infty} e^{-\lambda t}=1 / \lambda$

In a Weibull distribution, the result is not constant. Therefore, in order to propose a solvable model, we consider a constant MTBF as obtained from the exponential distribution. With this parameter, we can obtain the probability of it being in a reparation state given that it is not in maintenance using Eq. (51)

$P\left(R \mid M^{\prime}\right)=1-\frac{M T B F}{M T B F+t^{\text {repair }}}$

Where $t^{\text {repair }}$ is the time that it takes to repair the unit. This result is intuitive, since if we consider any process equipment with a repair time that approximates to 0 , even if it fails a lot of times, it will never be in a repairing state since the reparation is instantaneous.

We introduce another set $n m \in N M$, as the ordered set of the number of maintenances (52).

$N M:=\{n m:$ Set of the possible number of maintenances in a year $\}$

The main parameter dependent on this set is the number of maintenances, which is defined as shown in Eq. (53).

$N_{n m}^{\text {main }}=n m-1 \quad \forall n m \in N M$

If we have parameters for the time that takes to perform a maintenance in a unit $j$, at a stage $k$, called $t_{k, j}^{\text {main }}$, we can define the time taken to perform a number of maintenances in a stage, considering a setting, for a unit, as shown in Eq. (54).

$T M_{n m, k, s, j}= \begin{cases}t_{k, j}^{\text {main }} N_{n m}^{\text {main }} & \forall k, s, j \in M M_{k, s, j}, n m \in N M \\ 0 & \forall k, s, j \notin M M_{k, s, j}, n m \in N M\end{cases}$

Similar to Eq. (30), now we calculate the operation time of each machine for each setting. Now we consider the maintenance time, as shown in Eq. (55).

$t_{n m, k, s, j}^{o p}=\left[t^{\text {total }}-T M_{n m, k, s, j}-\sum_{\substack{j^{\prime}<j \\ j^{\prime} \in J S K_{k, s, j} \\ j^{\prime} \in J F_{k, j}}} t_{n m, k, s, j^{\prime}}^{o p}\right]\left(1-P\left(R \mid M^{\prime}\right)_{k, j}\right)$

$\forall n m \in N M, \forall k, s, j \in J S K_{k, s, j}, J F_{j, k}$

$t_{n m, k, s, j}^{o p}=\left[t^{\text {total }}-T M_{n m, k, s, j}-\sum_{\substack{j^{\prime} \in J S K_{k, s, j} \\ j^{\prime} \in J F_{k, j}}} t_{n m, k, s, j^{\prime}}^{o p}\right]\left(1-P\left(R \mid M^{\prime}\right)_{k, j}\right)$

$\forall n m \in N M, \forall k, s, j \in J S K_{k, s, j}, J H_{j, k}, J 1_{j, k}$

$\left.\begin{array}{l}t_{n m, k, s, j}^{o p}=\left[t^{\text {total }}-T M_{n m, k, s, j}-\sum_{\substack{j^{\prime} \in J S K_{k, s, j} \\ j^{\prime} \in J_{k, j}}} t_{n m, k, s, j^{\prime}}^{o p}\right. \\ \forall n m \in N M, \forall k, s, j \in J S K_{k, s, j}, J H_{j, k}, J 2_{j, k}\end{array}\right] \frac{N H_{k, s}-1}{N S_{k, s}}\left(1-P\left(R \mid M^{\prime}\right)_{k, j}\right)$

Now we have the operation time of each machine for each setting. By using this time in Eq. (38) we can obtain the probability of having at least a failure during the year. The equations, which depends on the number of maintenances, which stage, which setting and at which unit we are looking, is defined by Eq. (56). 


$$
\begin{gathered}
p f_{n m, k, s, j}^{\text {exponential }}= \begin{cases}1-\left[\exp \left(-\lambda_{k, j} \frac{t_{n m, k, j, j}^{o p}}{N_{n m}^{m \text { ain }}+1}\right)\right]^{N_{n m}^{m m i n}+1} & \forall n m \in N M, k, s, j \in M M_{k, s, j} \\
1-\exp \left(-\lambda_{k, j} t_{n m, k, s, j}^{o p}\right) & \forall n m \in N M, k, s, j \notin M M_{k, s, j}\end{cases} \\
p f_{n m, k, s, j}^{\text {Weibull }}= \begin{cases}1-\left[\exp \left(-\left(\frac{t_{n m, k, s, j}^{o p}}{N_{n m}^{\text {mal }}+1} \frac{1}{\theta_{k, j}}\right)^{k}\right)\right]^{N_{n m}^{\text {main }}+1} & \forall n m \in N M, k, s, j \in M_{k, s, j} \\
1-\exp \left(-\left(\frac{t_{n m, k, s, j}^{o p}}{\theta_{k, j}}\right)^{k}\right) & \forall n m \in N M, k, s, j \notin M M_{k, s, j}\end{cases}
\end{gathered}
$$

We can establish how to obtain the general availability of the unit. It follows Eq. (57).

$$
\begin{aligned}
p a_{n m, k, s, j}=\frac{t^{\text {total }}-T M_{n m, k, s, j}}{t^{\text {total }}} & \left(1-P\left(R \mid M^{\prime}\right)_{k, j}\right) \\
& \forall n m \in N M, k, s, j \in J S K_{k, s, j}
\end{aligned}
$$

A direct intuition would be to use this general availability in an equation similar to Eq. (10). Certainly, it would be the first and more general idea. However, we would incur some overestimations of the probability of not working at half capacity. First, we define the probability of being in a maintenance state and the probability of being in a repairing state, as shown in Eq. (58).

$P(M)_{n m, k, s, j}=\left\{\begin{array}{lr}T M_{n m, k, s, j} / t^{\text {total }} & \forall n m \in N M, k, s, j \in M M_{k, s, j}, J S K_{k, s, j} \\ 0 & \forall n m \in N M, k, s, j \in J S K_{k, s, j} \notin M M_{k, s, j}\end{array}\right.$
$P(R)_{n m, k, s, j}=\left(1-P(M)_{n m, k, s, j}\right) P\left(R \mid M^{\prime}\right)_{k, j} \quad \forall n m \in N M, k, s, j \in J S K_{k, s, j}$

Now, if we consider a setting in a stage where there is only one machine labeled as main machine, the equation to obtain the probability of that stage working at half capacity would be exactly analogous to Eq. (10), where the probability of being available would be obtained from Eq. (57). This is due to the fact that, between different machines, the states of failure and maintenance are not exclusive.

To illustrate this, consider the following example. We are in a setting where we have one full capacity machine and one half capacity machine. We call them $J$ and $j$ respectively. The main machine is, by the definition of the set above, the full capacity machine $J$. In order to work at half capacity, the main machine must be in a not working state. To obtain the probability of being in a not working state for the first machine, and knowing that for that machine the maintenance and repairing states are exclusive, we use Eq. (59).

$$
\begin{aligned}
P(W)_{J} & =1-\left(P(M)_{J}+P(R)_{J}\right)=1-\frac{T M_{J}}{t^{\text {total }}}-P\left(R \mid M^{\prime}\right) \frac{t^{\text {total }}-T M_{J}}{t^{\text {total }}} \\
& =p a_{J}
\end{aligned}
$$

So the probability of the main machine not working is given above. In order to work at half capacity, the not-main machine $j$ must be working, so its probability is as shown in Eq. (60).

$P(W)_{j}=1-\left(P(M)_{j}+P(R)_{j}\right)=1-0-P(R \mid \neg M) \times 1=p a_{j}$

Therefore, for half capacity, we have the following probabilities shown in Eq. (61).

$$
\begin{aligned}
P^{H} & =P\left(M_{J} \cap W_{j}\right)+P\left(R_{J} \cap W_{j}\right)=P(M)_{J} p a_{j}+P(R)_{J} p a_{j}= \\
& =\left(P(M)_{J}+P(R)_{J}\right) p a_{j}=\left(1-p a_{J}\right) p a_{j}
\end{aligned}
$$

Again, this was the case before, where we assumed that between the two machines there is not any mutually exclusive probability. However, when we have more than one main machine, and therefore, more than one maintenance at a machine, the coherent way of thinking is to consider the following rules:

1- The states of maintenance of the two machines are not performed at the same time. Therefore, they are mutually exclusive, but not exhaustive.
2- The states of repairing are not mutually exclusive, nor are mutually exclusive between the two machines. If one machine is in maintenance, the other one may require reparation. However, they still are mutually exclusive with the maintenance state for the same machine, in that it cannot be at the same time in a state of repairing and maintenance.

The simplest example for this would be a setting where there are only two half capacity machines, $j 1, j 2$. If there is a full capacity machine, automatically it is the only main machine. However, we considered that for settings without full capacity machines, all the half capacity machines are susceptible to have maintenance, and are therefore main machines. The full space of possibilities is shown in Eq. (62).

$$
\begin{aligned}
S= & \left\{W_{j 1} W_{j 2}, W_{j 1} M_{j 2}, W_{j 1} R_{j 2}, M_{j 1} W_{2}, M_{j 1} M_{j 2}, M_{j 1} R_{j 2},\right. \\
& \left.R_{j 1} W_{j 2}, R_{j 1} M_{j 2}, R_{j 1} R_{j 2}\right\}
\end{aligned}
$$

We are working with the assumption of not allowing the two maintenances to take place at the same time, therefore the reduced space of probabilities is as shown in Eq. (63).

$S^{\prime}=1-P\left(M_{j 1} \cap M_{j 2}\right)$

We will have to scale the rest of probabilities to this reduced space of probabilities. Now, if we check from the whole space of probabilities which settings result in working at half capacity, we get Eq. (64).

$P^{H}=\frac{P\left(W_{j 1} \cap M_{j 2}\right)+P\left(W_{j 1} \cap R_{j 2}\right)+P\left(M_{j 1} \cap W_{j 2}\right)+P\left(R_{j 1} \cap W_{j 2}\right)}{S^{\prime}}$

The general equation that defines the numerator of the half capacity probability is shown in Eq. (65).

$P^{H}=\sum_{j}\left(\sum_{m \neq j} P(M)_{m} P\left(W_{j}\right) \prod_{\substack{l \neq j \\ l \neq m}} P(F)_{l}+P\left(W_{j}\right) \prod_{m \neq j} P(F)_{m}\right)$

The probability of working at full capacity is also changed from the one previously presented in Eq. (13). The main idea remains the same, to deduct from the total probability the probability of total failure plus the probability of working at half capacity. However, the full failure equation now takes a different form, as shown in Eq. (66).

$P^{F}=1-\frac{\left(\sum_{j} P(M)_{j} \prod_{l \neq j} P(F)_{l}+\prod_{j} P(F)_{j}\right)}{S^{\prime}}-P^{H}$

Unfortunately, the reduced space of probabilities $S^{\prime}$ has not a direct and easy equation that can be produced for all the cases with multiple main machines, at least not without creating another set of combinations. Therefore, we develop the formulation for cases when there are two and three main machines simultaneously. These are shown in Eq. (67).

$$
\begin{aligned}
& S^{\prime} \text { (twomachines) : } S^{\prime}=1-\prod_{j} P(M)_{j} \\
& S^{\prime} \text { (threemachines) : } \\
& \quad S^{\prime}=1-\left(\sum_{j}\left(P(W)_{j}+P(R)_{j}\right) \prod_{j^{\prime} \neq j} P(M)_{j^{\prime}}+\prod_{j} P(M)_{j}\right)
\end{aligned}
$$

We define two more sets, as the set of settings with two main machines and the set of settings with three main machines (68).

$M M 2_{k, s}:=\{(k, s):$ There are two main machines in the setting $s$ at the stage $k\}$

$M M 3_{k, s}:=\{(k, s)$ : There are three main machines in the setting $s$ at the stage $k\}$ 
These sets can be dynamically defined using Eq. (69):

$\begin{aligned} M M 2_{k, s} & =\left\{(k, s) \mid \sum_{j \in M M_{k, s, j}} 1=2\right\} \\ M M 3_{k, s} & =\left\{(k, s) \mid \sum_{j \in M M_{k, s, j}} 1=3\right\}\end{aligned}$

Since we introduced a set of the number of maintenances, we require a new binary variable. We define $w_{n m, k, s}$ as the binary variable that has a value of 1 when in the stage $k$, setting $s$, we are in the number of maintenances defined by $\mathrm{nm}$. We can only be in a number of maintenances at the same time, as defined by Eq. (70).

$z_{k, s}=\sum_{n m \in N M} w_{n m, k, s} \quad \forall k, s \in K S_{k, s}$

We define two new variables, $P n m_{n m, k, s}^{H}, P n m_{n m, k, s}^{F}$, as the probability of a stage $k$, setting $s$ with a number of maintenances $n m$ of working at half and full capacity respectively. The probabilities of working at half capacity are obtained by Eq. (71).

$P n m_{n m, k, s}^{H}=\left(\prod_{j \in J F_{j, k}, J S K_{k, s, j}}\left(1-p a_{n m, k, s, j}\right) \sum_{j \in J H_{j, k}, J S K_{k, s, j}} p a_{n m, k, s, j} \prod_{\substack{l \neq j \\ l \in J H_{j, k}, J S K_{k, s, j}}}\left(1-p a_{n m, k, s, j}\right)\right) w_{n m, k, s}$

$\forall n m \in N M, k, s \in K S_{k, s} \notin M M 2_{k, s} \notin M M 3_{k, s}$

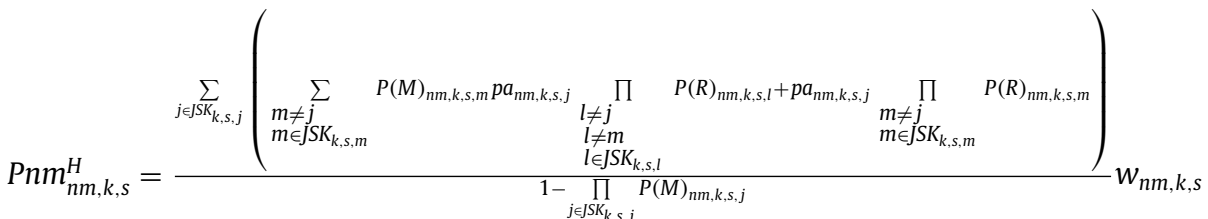

$\forall n m \in N M, k, s \in M M 2_{k, s}$

From these we define the probability of working at half capacity and full capacity respectively for a stage $k$, setting $s$, as Eq. (73).

$P s_{k, s}^{H}=\sum_{n m \in N M} P n m_{n m, k, s}^{H} \quad \forall k, s \in K S_{k, s}$

$P s_{k, s}^{F}=\sum_{n m \in N M} P n m_{n m, k, s}^{F} \quad \forall k, s \in K S_{k, s}$

And we can obtain the probability for a stage $k$ with Eqs. (12) and (14).

We have to account for the cost of the maintenances. These are given by Eq. (74).

$$
\begin{array}{ll}
C n m_{n m, k, s}^{\text {main }}=\left(\sum_{j \in M M_{k, s, j}} m c_{k, j}\right) N_{n m}^{\text {main }} W_{n m, k, s} & \forall n m \in N M, k, s \in K S_{k, s} \\
C s_{k, s}^{\text {main }}=\sum_{n \in \in N M} C n m_{n m, k, s}^{\text {main }} & \forall k, s \in K S_{k, s} \\
C_{k}^{\text {main }}=\sum_{s \in K S_{k s}} C S_{k, s}^{\text {main }} & \forall k \in K
\end{array}
$$

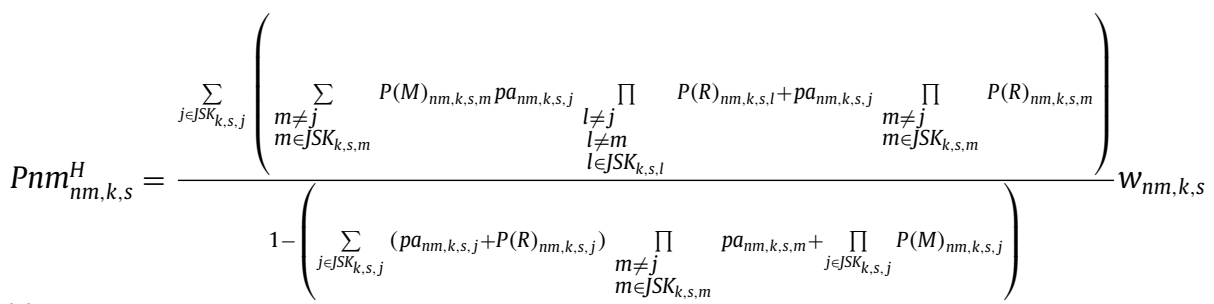

$\forall n m \in N M, k, s \in M M 3_{k, s}$

The full capacity probabilities are shown in Eq. (72).

$\operatorname{Pnm}_{n m, k, s}^{F}=\left(1-\prod_{j \in J F_{j, k}, J S K_{k, s, j}}\left(1-p a_{n m, k, s, j}\right) \prod_{j \in J H_{j, k}, J S K_{k, s, j}}\left(1-p a_{n m, k, s, j}\right)-P n m_{n m, k, s}^{H}\right) w_{n m, k, s}$

$\forall n m \in N M, k, s \in K S_{k, s} \notin M M 2_{k, s} \notin M M 3_{k, s}$

$P n m_{n m, k, s}^{F}=\left(1-\frac{\sum_{j \in S K_{k, s, j}} P(M)_{n m, k, s, j} \prod_{\substack{l \neq j \\ l \in J S K_{k, s, l}}} P(R)_{n m, k, s, l}+\prod_{j \in J K_{k, s, j}} P(R)_{n m, k, s, j}}{1-\prod_{j \in S K_{k, s, j}} P(M)_{n m, k, s, j}}-P n m_{n m, k, s}^{H}\right) W_{n m, k, s}$

$\forall n m \in N M, k, s \in M M 2_{k, s}$

$P n m_{n m, k, s}^{F}=\left(1-\frac{\sum_{j \in J K_{k, s, j}} P(M)_{n m, k, s, j} \prod_{\substack{l \neq j \\ l \in J S K_{k, s, l}}} P(R)_{n m, k, s, l}+\prod_{j \in J K_{k, s, j}} P(R)_{n m, k, s, j}}{1-\left(\sum_{j \in S K_{k, s, j}}\left(p a_{n m, k, s, j}+P(R)_{n m, k, s, j}\right) \prod_{\substack{m \neq j \\ m \in J S K_{k, s, m}}} P(M)_{n m, k, s, m}+\prod_{j \in J K_{k, s, j}} P(M)_{n m, k, s, j}\right)}-P n m_{n m, k, s}^{H}\right) W_{n m, k, s}$

$\forall n m \in N M, k, s \in M M 3_{k, s}$ 


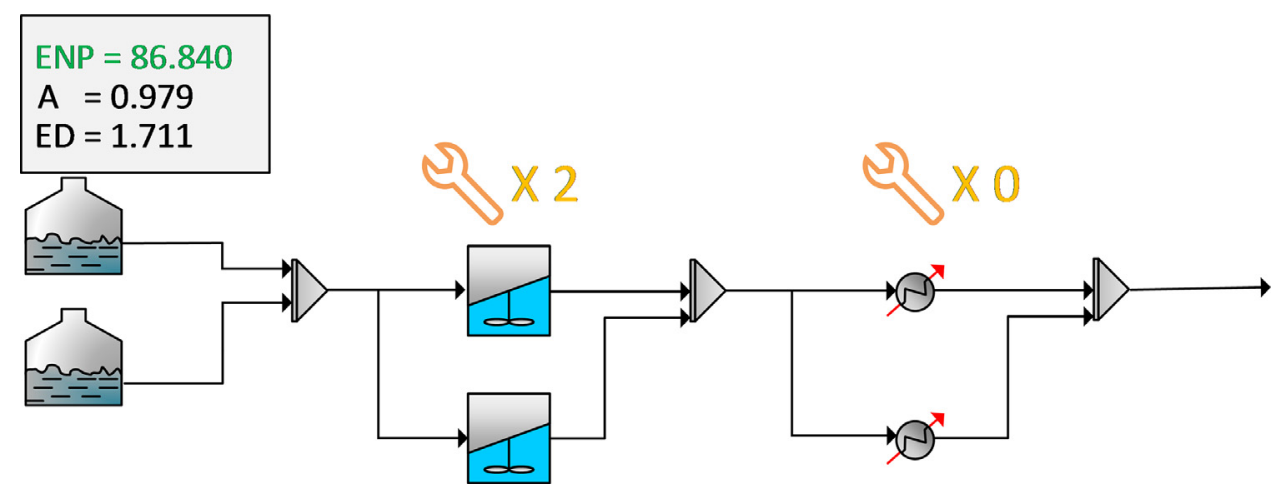

Fig. 13. Results of optimizing profit in the example problem including maintenance.

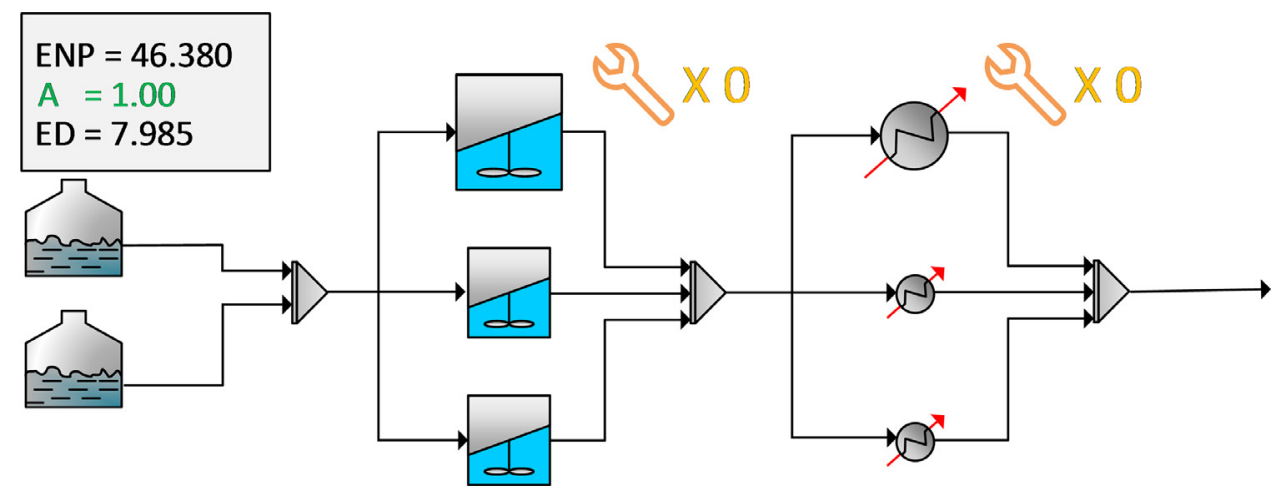

Fig. 14. Results of optimizing availability in the example problem including maintenance.

Where $m c_{k, j}$ is a parameter that specifies the maintenance cost of the unit $j$ in stage $k$. Thus, the new objective function is as shown in Eq. (75).

$$
E N P=E R V-P e n^{A}+B n^{A}-\sum_{k \in K}\left(C_{k}+C_{k}^{\text {main }}\right)-P^{S e n}{ }^{S} D
$$

Now, we repeat the example problem given in Fig. 3, but adding maintenance and using a Weibull distribution with $k=2$. We add the maintenance times and maintenance costs shown in Tables 3 and 4 . We also add a penalization in the objective function to the safety of $P e n^{S}=4$.

The results are shown in Figs. 13, 14 and 15.

We can extract some common observations. Once again, the result of maximizing the availability is the alternative where each stage has the setting full of machines. However, now we can notice that it also includes zero maintenances. This is expected, since the maintenances reduce the operation time and thus reduce the availability of the setting.

Table 3

Maintenance times for the example problem including maintenance.

\begin{tabular}{llll}
\hline Stage/Machine & Machine 1 & Machine 2 & Machine 3 \\
\hline Stage 1 & 48 & 46 & 46 \\
Stage 2 & 24 & 22 & 22 \\
\hline
\end{tabular}

Table 4

Maintenance costs for the example problem including maintenance.

\begin{tabular}{llll}
\hline Stage/Machine & Machine 1 & Machine 2 & Machine 3 \\
\hline Stage 1 & 0.15 & 0.12 & 0.12 \\
Stage 2 & 0.9 & 0.6 & 0.6 \\
\hline
\end{tabular}

\section{Assessment of the damage potential and the penalization to the objective function}

Assessing the damage potential of a unit is not an easy task. It is expected that it will depend on both the type of process unit (reactor, heat exchanger, distillation column, etc.) and the amount of chemical in that unit. It could be even argued that it will depend on the controllability of the unit, which results in a whole new layer of complications. Since this calculation is not considered inside the optimization model, it is not really important how nonconvex and non-linear it is. However, since we lack reliability and damage data enough to make a correct and complete assessment, in this paper we consider as the damage potential the damage radius obtained by the Dow's Fire and Explosion Index (AIChE, 1994) in meters. This is just a possibility, since the correct evaluation of the damage potential of a unit is out of the scope of this paper. There exist extensive methodologies used in quantitative risk assessment that define how to obtain individual, societal and various risk indices (AIChE, 2000). Since the objective of the paper is to showcase the capabilities of the model in assessing both the reliability and the safety of the design at an early stage, we consider the approximation of using the damage radius as adequate.

The penalization to the objective function presents another problem. The availability and the economic profit are easily related, since the expected revenue is just the annual revenue multiplied by the annual availability of the plant. However, the penalization of the damage is not as clear as a contract. There is no contractual agreement that allows a quantity of annual damage. Therefore, it is not clear how to systematically assign this penalization. There are tools that can help the user to define it, such as the Analytical Hierarchy Process (Saaty, 1990), which assign a value following the preferences of the decision-maker and result in a single objective function. In this work, we opt to consider safety and profit as completely separated objectives and perform a multi-objective 


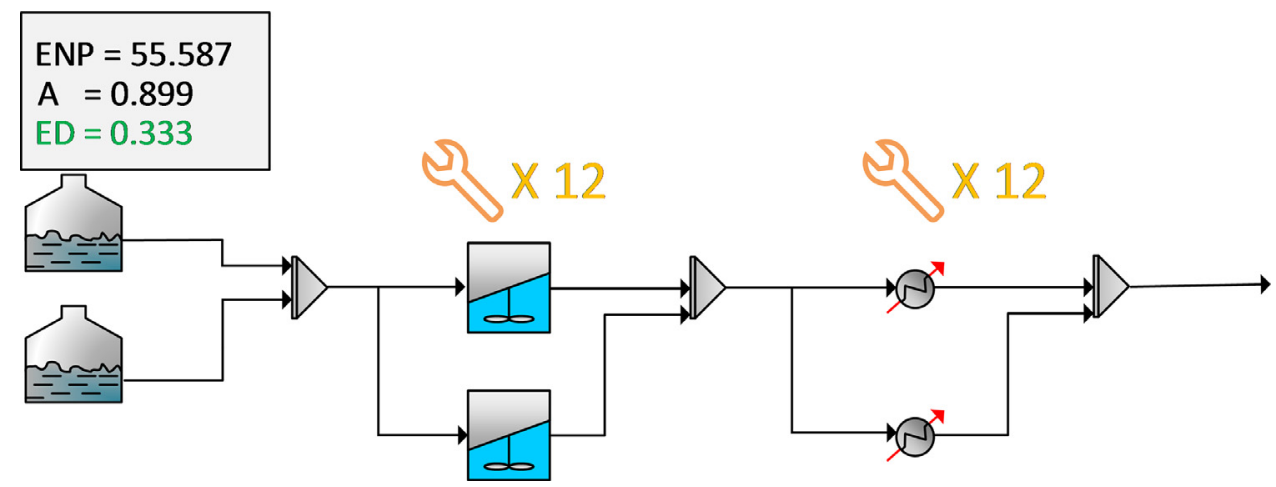

Fig. 15. Results of optimizing safety in the example problem including maintenance.

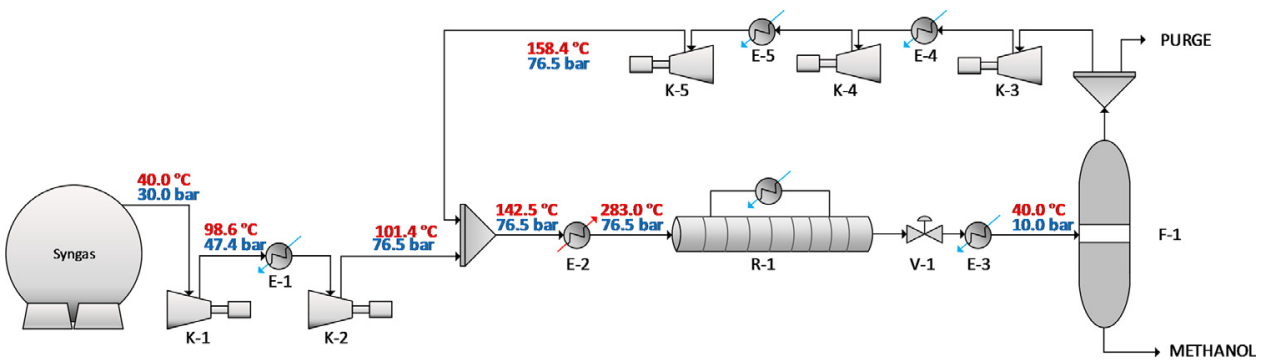

Fig. 16. Flowsheet of the case study.

optimization, which results in a Pareto frontier. How to deal with the multi-objective optimization problem is up to the decisionmaker, but some examples include objective reduction methods (Guillén-Gosálbez, 2011; Vázquez et al., 2018; Vázquez et al., 2018a, 2018b) or aggregated metrics, which are common in Life Cycle Analysis (M. Goedkoop et al., 1995; M. J. Goedkoop et al., 2009; Jolliet et al., 2003; PRé-Consultants, 2000). There are as well multiple other methods and new methodologies emerge continuously (Gupta et al., 2019; Safarzadeh et al., 2018; Y. Song and Teng, 2019) .In this work, we only provide the Pareto frontier and the final user, and the decision-maker should decide which is the chosen final design based on his/her preferences. However, during the case study, two points in the Pareto frontier are chosen to showcase the differences in the results of the model.

\section{Case study and results}

As a case study, we consider the methanol production plant, which has already been extensively studied (Abrol and Hilton, 2012; Luyben, 2010; Navarro-Amorós et al., 2014). Concretely, we focus on the work by Medrano et al. (2017), where the flowsheet was optimized both for the economic and environmental criteria. In this work, we consider the optimal economic flowsheet, which is shown in Fig. 16.

We consider the possibility of having two full capacity units and three half capacity units for each of the named process units of the flowsheet. We obtain the costs from Turton et al. (2012) and Ye et al. (2018), and the number of failures in a year from AIChE (1989). The parameters used in the model are shown in Table 5.

The maintenance cost is supposed as $1 \%$ of the unit cost. While this may seem low, we are considering a system where the process units tend to be durable but require small maintenances, such as cleaning the heat exchangers and the pipeline, whose major maintenance cost is the time that it is not operative.

We consider a superstructure with the following characteristics. The number of possible units of heat exchangers is two at full ca-
Table 5

Economic and safety data for the case study.

\begin{tabular}{|c|c|c|c|c|}
\hline Unit & Capacity & $\begin{array}{l}\text { Unit cost } \\
\text { (k\$/year) }\end{array}$ & $\begin{array}{l}\text { Damage } \\
\text { Radii (m) }\end{array}$ & $\begin{array}{l}\text { Failures } \\
\text { in } 10^{6} \mathrm{~h}\end{array}$ \\
\hline \multirow[t]{2}{*}{ E-1 } & $100 \%$ & 81.266 & 43.26 & 51.90 \\
\hline & $50 \%$ & 60.233 & 38.68 & 51.90 \\
\hline \multirow[t]{2}{*}{$\mathrm{E}-2$} & $100 \%$ & 414.98 & 47.82 & 51.90 \\
\hline & $50 \%$ & 227.09 & 46.56 & 51.90 \\
\hline \multirow[t]{2}{*}{ E-3 } & $100 \%$ & 852.25 & 40.74 & 51.90 \\
\hline & $50 \%$ & 445.73 & 39.54 & 51.90 \\
\hline \multirow[t]{2}{*}{ E-4 } & $100 \%$ & 166.53 & 43.93 & 51.90 \\
\hline & $50 \%$ & 102.87 & 42.00 & 51.90 \\
\hline \multirow[t]{2}{*}{ E-5 } & $100 \%$ & 173.09 & 45.72 & 51.90 \\
\hline & $50 \%$ & 106.15 & 43.79 & 51.90 \\
\hline \multirow[t]{2}{*}{$\mathrm{K}-1$} & $100 \%$ & 385.57 & 43.26 & 1430.00 \\
\hline & $50 \%$ & 231.56 & 38.68 & 1430.00 \\
\hline \multirow[t]{2}{*}{$\mathrm{K}-2$} & $100 \%$ & 401.76 & 44.15 & 1430.00 \\
\hline & $50 \%$ & 242.16 & 39.58 & 1430.00 \\
\hline \multirow[t]{2}{*}{$\mathrm{K}-3$} & $100 \%$ & 877.20 & 43.93 & 1430.00 \\
\hline & $50 \%$ & 569.82 & 42.00 & 1430.00 \\
\hline \multirow[t]{2}{*}{$\mathrm{K}-4$} & $100 \%$ & 901.70 & 45.72 & 1430.00 \\
\hline & $50 \%$ & 587.43 & 43.79 & 1430.00 \\
\hline \multirow[t]{2}{*}{ K-5 } & $100 \%$ & 947.56 & 47.16 & 1430.00 \\
\hline & $50 \%$ & 620.55 & 45.23 & 1430.00 \\
\hline V-1 & $100 \%$ & 1.00 & 45.02 & 1.00 \\
\hline \multirow[t]{2}{*}{$\mathrm{R}-1$} & $100 \%$ & 826.88 & 54.02 & 52.00 \\
\hline & $50 \%$ & 436.85 & 52.58 & 52.00 \\
\hline \multirow[t]{2}{*}{$\mathrm{F}-1$} & $100 \%$ & 549.66 & 45.78 & 0.0745 \\
\hline & $50 \%$ & 286.76 & 45.00 & 0.0745 \\
\hline
\end{tabular}

pacity and two at half capacity. The number of possible units of $\mathrm{V}-1$ is three at full capacity. For the rest of the units, we consider the possibility of one unit at full capacity and two units at half capacity. The number of possible annual maintenances is 12 , one per month.

The data of the availabilities with zero maintenances, this meaning, considering only the repair state, is shown in Table 6 .

The data of the maintenance times for the units is shown in Table 7. 


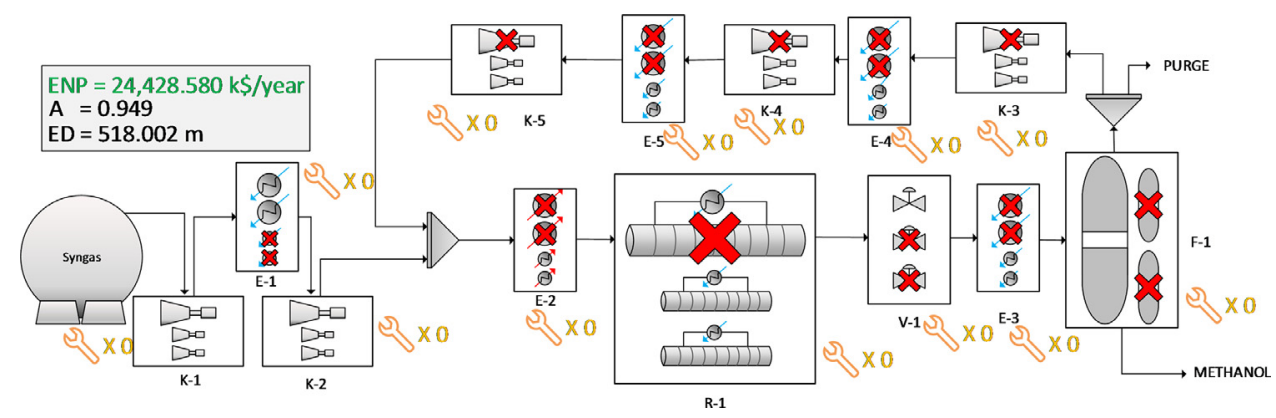

Fig. 17. Optimal ENP flowsheet of the case study.

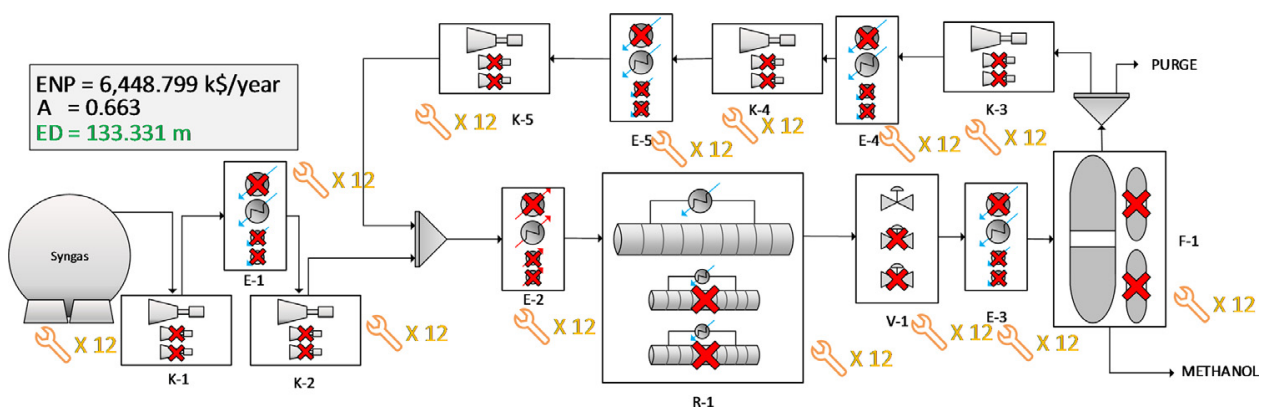

Fig. 18. Optimal safety flowsheet of the case study.

Table 6

Availabilities without considering maintenances for the case study.

\begin{tabular}{lllll}
\hline Stage & $\mathrm{j}_{1}$ & $\mathrm{j}_{2}$ & $\mathrm{j}_{3}$ & $\mathrm{j}_{4}$ \\
\hline E-1 to E-5 & 0.9975 & 0.9973 & 0.9988 & 0.9986 \\
K-1 to K-5 & 0.9722 & 0.9859 & 0.9845 & - \\
V-1 & 0.9999 & 0.9999 & 0.9999 & - \\
R-1 & 0.9948 & 0.9974 & 0.9974 & - \\
F-1 & 0.9999 & 0.9999 & 0.9999 & - \\
\hline
\end{tabular}

Table 7

Maintenance times for the units in the case study [h].

\begin{tabular}{lllll}
\hline Stage & $\mathrm{j}_{1}$ & $\mathrm{j}_{2}$ & $\mathrm{j}_{3}$ & $\mathrm{j}_{4}$ \\
\hline E-1 to E-5 & 14.40 & 15.60 & 7.20 & 7.80 \\
K-1 to K-5 & 6.00 & 3.00 & 3.30 & - \\
V-1 & 0.30 & 0.30 & 0.30 & - \\
R-1 & 30.00 & 15.00 & 18.00 & - \\
F-1 & 27.00 & 13.50 & 15.00 & - \\
\hline
\end{tabular}

Table 8

Parameters for the case study.

\begin{tabular}{lllll}
\hline Revenue & fpen $^{\mathrm{A}}$ & fben $^{\mathrm{A}}$ & $\mathrm{A}^{\text {low }}$ & $\mathrm{A}^{\text {high }}$ \\
\hline $33834.90 \mathrm{k} \$$ & $30000.00 \mathrm{k} \$$ & $30000.00 \mathrm{k} \$$ & 0.950 & 0.970 \\
\hline
\end{tabular}

Table 9

Results of the case study without considering optimization.

\begin{tabular}{lll}
\hline ENP & A & Damage radius \\
\hline $19391.749 \mathrm{k} \$$ & 0.853 & $317.784 \mathrm{~m}$ \\
\hline
\end{tabular}

The time window considered is a year, with 8000 hours of expected work. The rest of the parameters are shown in Table 8.

The distribution considered is a Weibull distribution with $k=2$. The results for the simple flowsheet with only one main full capacity unit without maintenances is shown in Table 9.

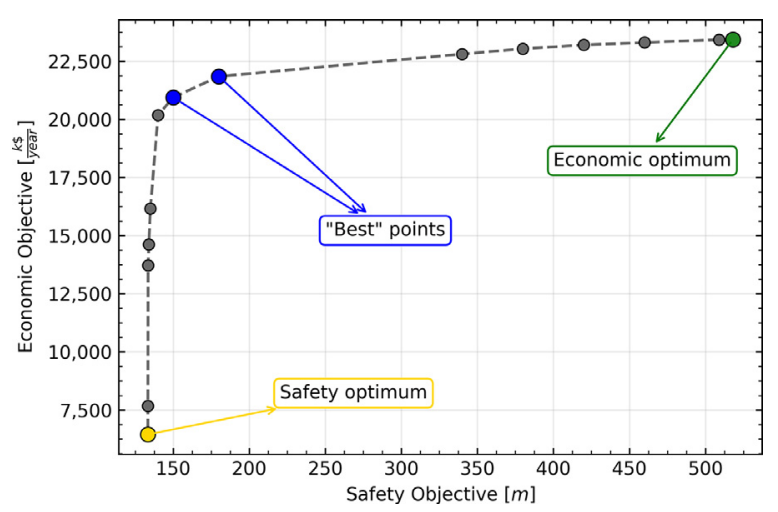

Fig. 19. Pareto front from the multi-objective optimization.

The model optimizing the expected net profit consists of 10,543 equations and 1,925 discrete variables. It was solved in $13.0 \mathrm{~s}$ using SCIP (Achterberg, 2009). The resultant flowsheet is shown in Fig. 17.

The model optimizing the safety was solved in $1.00 \mathrm{~s}$ using SCIP. The resultant flowsheet is shown in Fig. 18.

As it is expected, the safest flowsheet consists of the highest allowed number of maintenances. The availability is then greatly reduced, since the higher the number of maintenances the lesser the time of operation available at a given year. It chooses the minimum number of units, and the reason that in the cases of the heat exchangers the second heat exchanger is chosen is due to the fact that in Table 6 we can see that we penalize a small amount the availability for the second unit of each capacity. The most economic flowsheet has not any maintenances, since they incur a cost and they reduce the annual availability of the unit, which has repercussions in the expected net profit.

However, from the results in the economic and safety objectives, it is apparent that there is room for many additional middle solutions. Since the solving time of the model is not too high, we perform a multi-objective optimization using the augmented 


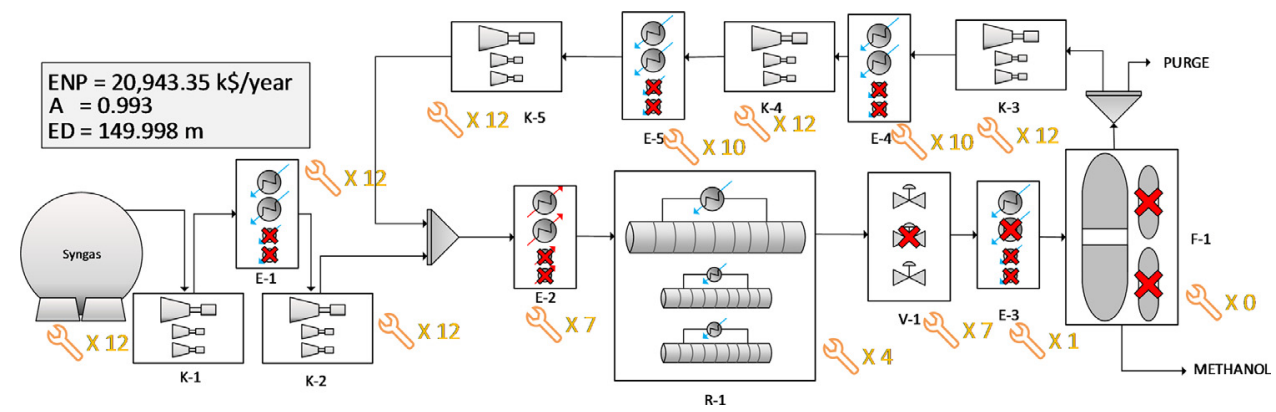

Fig. 20. Pareto flowsheet of the first chosen intermediate point.

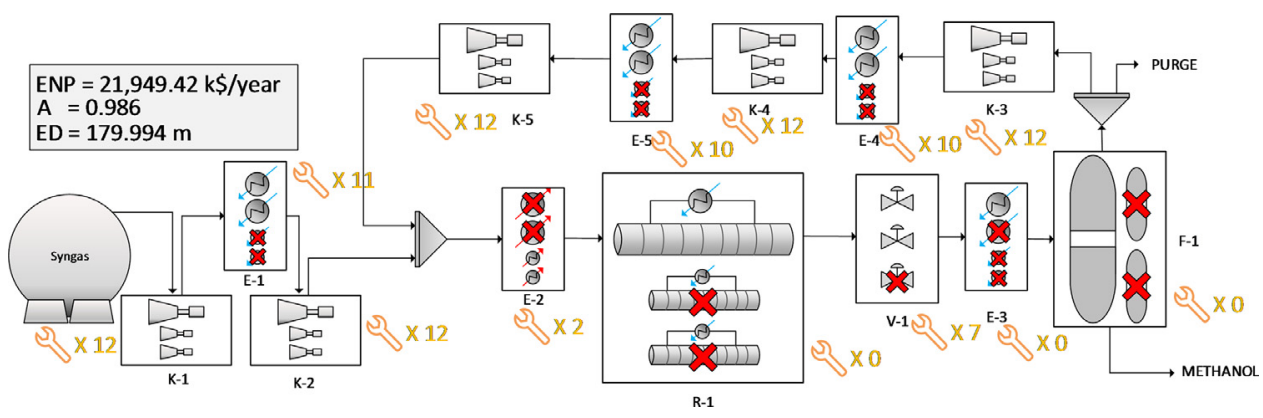

Fig. 21. Pareto flowsheet of the second chosen intermediate point.

$\varepsilon$-constraint method (Mavrotas, 2009; Mavrotas and Florios, 2013). The resultant Pareto frontier is shown in Fig. 19.

From that figure, we choose two points in order to showcase their resultant flowsheet. These are not necessarily the best points, since in a Pareto front all the points are mathematically equally good. However, we consider that looking at the slopes of the front before and after these points, we can highlight these two solutions. These are shown in Figs. 20 and 21.

\section{Conclusions}

The paper presents an MINLP model able to choose designs in a superstructure of potential process units. The model presents a safety risk analysis, based on the damage potential that certain units may incur and the probability of failure of those units for a certain period of time. It also takes into account the availability of the units, considering the repair times and the failure rates.

In addition, the model introduces the possibility of a set number of maintenances equally spaced in the time period chosen. Maintenances are supposed to reduce the probability of failure of a process unit in a set interval of time, but their inclusion reduces the availability of said process units and increases the costs associated with the flowsheet. Small examples illustrate the steps of the model, before presenting the case study and the ability of the model to optimize the flowsheet superstructure. A Pareto frontier considering the objectives of expected profit and expected safety is presented, and the availability is interweaved in these two objectives, since it affects both.

The results showcase the effectivity of the model in producing the optimal result regarding the safety and economic objectives, while still considering the availability as an important factor. Future work can be done exploring the development of a similar model and interweaving it with Markov chains, which are a common approach in reliability engineering.

\section{Declaration of Competing Interest}

"The author(s) declare(s) that there is no conflict of interest regarding the publication of this article."

\section{CRediT authorship contribution statement}

Daniel Vázquez: Conceptualization, Data curation, Formal analysis, Investigation, Methodology, Software, Writing - original draft, Writing - review \& editing. Rubén Ruiz-Femenia: Software, Supervision, Validation, Funding acquisition, Writing - review \& editing. José A. Caballero: Formal analysis, Funding acquisition, Project administration, Resources, Supervision, Validation, Writing - review \& editing.

\section{Acknowledgments}

The authors acknowledge financial support from the Spanish "Ministerio de Economía, Industria y Competitividad" (CTQ201677968-C3-2-P, AEI/FEDER, UE) and from the "Generalitat Valenciana" (PROMETEO/2020/064).

\section{References}

Abrol, S., Hilton, C.M., 2012. Modeling, simulation and advanced control of methanol production from variable synthesis gas feed. Comput. Chem. Eng. 40, 117-131.

Achterberg, T., 2009. SCIP: solving constraint integer programs. Math. Program. Comput. 1, 1-41.

AIChE, 1989. Guidelines for Process Equipment Reliability Data With Data Tables. Center for Chemical Process Safety of the American Institute of Chemical Engineers: American Institute of Chemical Engineers. Center for Chemical Process Safety.

AIChE, 1994. Dow's Fire \& Explosion Index Hazard Classification Guide.

AIChE, 2000. Guidelines for chemical process quantitative risk analysis. Center for Chemical Process Safety of the American Institute of Chemical Engineers, 2nd ed. Wiley-Interscience.

AIChE, 2010. Inherently Safer Chemical Processes: A Life Cycle Approach. Wiley.

Al-Mutairi, E.M., Suardin, J.A., Mannan, M.S., El-Halwagi, M.M., 2008. An optimization approach to the integration of inherently safer design and process scheduling. J. Loss Prev. Process Ind. 21, 543-549.

Goedkoop, M., Demmers, M., Collignon, M., 1995. The Eco-indicator 95: weighting method for environmental effects that damage ecosystems or human health on a European Scale. Contains 100 Indicators for Important Materials and Processes. Manual for designers. Novem. 
Goedkoop, M.J., Heijungs, R., De Schyver, A., Struijs, J., Van Zelm, R., 2009. ReCiPe 2008, A Life Cycle Impact Assessment Method Which Comprises Harmonised Category Indicators at The Midpoint and The Endpoint Level.

Goel, H., Grievink, J., Herder, P., \& Weijnen, M. (2003). Optimal Reliability Design Of Process Systems At The Conceptual Stage Of Design.

Goel, H.D., Grievink, J., Weijnen, M.P.C., 2003. Integrated optimal reliable design, production, and maintenance planning for multipurpose process plants. Comput. Chem. Eng. 27, 1543-1555.

Grossmann, I.E., Trespalacios, F., 2013. Systematic modeling of discrete-continuous optimization models through generalized disjunctive programming. AIChE J. 59, 3276-3295.

Guillén-Gosálbez, G., 2011. A novel MILP-based objective reduction method for multi-objective optimization: Application to environmental problems. Comput. Chem. Eng. 35, 1469-1477.

Gupta, S., Soni, U., Kumar, G., 2019. Green supplier selection using multi-criterion decision making under fuzzy environment: a case study in automotive industry. Comput. Ind. Eng. 136, 663-680.

Houssin, R., Coulibaly, A., 2014. Safety-based availability assessment at design stage. Comput. Ind. Eng. 70, 107-115.

Jafari, M.J., Mohammadi, H., Reniers, G., Pouyakian, M., Nourai, F., Torabi, S.A., Rafiee Miandashti, M., 2018. Exploring inherent process safety indicators and approaches for their estimation: a systematic review. J. Loss Prev. Process Ind. $52,66-80$.

Jolliet, O., Margni, M., Charles, R., Humbert, S., Payet, J., Rebitzer, G., Rosenbaum, R. 2003. IMPACT 2002+: A new life cycle impact assessment methodology. Int. J. Life Cycle Assess. 8, 324

Khan, F.I., Abbasi, S.A., 1998. Multivariate Hazard identification and ranking system. In: Process Saf. Progr., p. 17.

Khan, F.I., Husain, T., Abbasi, S.A., 2001. Safety weighted hazard index (SWeHI). Process Saf. Environ. Prot. 79, 65-80.

Kletz, T., Amyotte, P., 2010. A Handbook for Inherently Safer Design, Second Edition ed..

Kondili, E., Pantelides, C.C., Sargent, R.W.H., 1993. A general algorithm for short-term scheduling of batch operations-I. MILP formulation. Comput. Chem. Eng. 17, 211-227.

Lee, S., Grossmann, I.E., 2000. New algorithms for nonlinear generalized disjunctive programming. Comput. Chem. Eng. 24, 2125-2141.

Lin, Z., Zheng, Z., Smith, R., Yin, Q., 2012. Reliability issues in the design and optimization of process utility systems. Theor. Found. Chem. Eng. 46, 747-754.

Lisnianski, A., Frenkel, I., Ding, Y., 2010. Multi-state System Reliability Analysis and Optimization for Engineers and Industrial Managers. Springer, London.

Luyben, W.L., 2010. Design and control of a methanol reactor/column process. Ind. Eng. Chem. Res. 49, 6150-6163.

Mavrotas, G., 2009. Effective implementation of the $\varepsilon$-constraint method in Multi-Objective Mathematical Programming problems. Appl. Math. Comput. 213, 455-465.

Mavrotas, G., Florios, K., 2013. An improved version of the augmented $\varepsilon$-constraint method (AUGMECON2) for finding the exact pareto set in multi-objective integer programming problems. Appl. Math. Comput. 219, 9652-9669.

Medrano, J.D., Ruiz-Femenia, R., Caballero, J.A., 2017. Multi-objective optimization of a methanol synthesis process superstructure with two-step carbon dioxide consumption. Comput. Aided Chem. Eng. 40, 721-726.
Navarro-Amorós, M.A., Ruiz-Femenia, R., Caballero, J.A., 2014. Integration of modular process simulators under the Generalized Disjunctive Programming framework for the structural flowsheet optimization. Comput. Chem. Eng. 67, 13-25.

Ordouei, M.H., Elkamel, A., Al-Sharrah, G., 2014. New simple indices for risk assessment and hazards reduction at the conceptual design stage of a chemical process. Chem. Eng. Sci. 119, 218-229.

Pistikopoulos, E.N., Vassiliadis, C.G., Arvela, J., Papageorgiou, L.G., 2001. Interactions of maintenance and production planning for multipurpose process plantsa system effectiveness approach. Ind. Eng. Chem. Res. 40, 3195-3207.

PRé-Consultants, 2000. The Eco-Indicator 99: A Damage Oriented Method For Life Cycle Impact Assessment, Methodology Report And Manual For Designers. Pré Consultants, Amersfoort, The Netherlands.

Roy, N., Eljack, F., Jiménez-Gutiérrez, A., Zhang, B., Thiruvenkataswamy, P., El-Halwagi, M., Mannan, M.S., 2016. A review of safety indices for process design. Curr Opin. Chem. Eng. 14, 42-48.

Ruiz-Femenia, R., Fernández-Torres, M.J., Salcedo-Díaz, R., Gómez-Rico, M.F., Caballero, J.A., 2017. Systematic tools for the conceptual design of inherently safer chemical processes. Ind. Eng. Chem. Res. 56, 7301-7313.

Rusli, R., Shariff, A.M., Khan, F.I., 2013. Evaluating hazard conflicts using inherently safer design concept. Saf. Sci. 53, 61-72.

Saaty, T.L., 1990. Decision Making for Leaders: The Analytic Hierarchy Process for Decisions in a Complex World. University of Pittsburgh.

Safarzadeh, S., Khansefid, S., Rasti-Barzoki, M., 2018. A group multi-criteria decision-making based on best-worst method. Comput. Ind. Eng. 126, 111-121.

Sharda, B., \& Bury, S. (2008). A discrete event simulation model for reliability modeling of a chemical plant.

Song, D., Yoon, E.S., Jang, N., 2018. A framework and method for the assessment of inherent safety to enhance sustainability in conceptual chemical process design. J. Loss Prev. Process Ind. 54, 10-17.

Song, Y., Teng, C., 2019. Optimal decision model and improved genetic algorithm for disposition of hierarchical facilities under hybrid service availability. Comput. Ind. Eng. 130, 420-429.

Turton, R., Bailie, R.C., Whiting, W.B., Shaeiwitz, J.A., Bhattacharyya, D., 2012. Analysis, Synthesis, and Design of Chemical Processes, 4th ed. Prentice Hall.

Vázquez, D., Fernández-Torres, M.J., Ruiz-Femenia, R., Jiménez, L., Caballero, J.A. 2018. MILP method for objective reduction in multi-objective optimization. Comput. Chem. Eng. 108, 382-394.

Vázquez, D., Ruiz-Femenia, R., Caballero, J.A., 2019. OFISI, a novel optimizable inherent safety index based on fuzzy logic. Comput. Chem. Eng., 106526.

Vázquez, D., Ruiz-Femenia, R., Jiménez, L., Caballero, J.A., 2018a. MILP models for objective reduction in multi-objective optimization: error measurement considerations and non-redundancy ratio. Comput. Chem. Eng. 115, 323-332.

Vázquez, D., Ruiz-Femenia, R., Jiménez, L., Caballero, J.A., 2018b. Multiobjective early design of complex distillation sequences considering economic and inherent safety criteria. Ind. Eng. Chem. Res. 57, 6992-7007.

Ye, Y., Grossmann, I.E., Pinto, J.M., 2018. Mixed-integer nonlinear programming models for optimal design of reliable chemical plants. Comput. Chem. Eng. 116, 3-16.

Yi, K., Xiao, H., Kou, G., Peng, R., 2019. Trade-off between maintenance and protection for multi-state performance sharing systems with transmission loss. Comput. Ind. Eng. 136, 305-315.

Zio, E., 2009. Reliability engineering: old problems and new challenges. Reliab. Eng. Syst. Saf. 94, 125-141. 